\title{
Comportement et stabilité des régulateurs contenant des éléments à caractéristique non linéaire
}

\author{
(Précédé d'un aperçu de la méthode de Nyquist et des méthodes dérivées \\ appliquées à l'hydraulique.)
}

\section{Behaviour and stability of governors with elements of non-linear characteristics}

(Preceeded by an outline of the Nyquist method and methods derived from it applied to hydraulics.)

PAR R. MIEYER

INGENEUR AUX FTALISSENENTS NEYRPIC:

(Voir la Houille Blanche, $\mathrm{n}^{\circ} 3,1952$ p. 394 et $\mathrm{n}^{\circ} 5,1952$, p. 665)

Etude sommaire de quelques exemples d'installations contenant des éléments non linéaires pour illuslser la méthode d'étude décrite dans

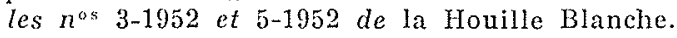
Comparaison des résultats avec ceux obtenus par ailleurs (dans les quelques cas où de tels résultats existaient jusqu'd présent).

Plusieurs points sont prorticulièrement à noter:

1. Etude d'un régulateur par tout ou rien accélérotachymétrique de conception simple: l'adjonction d'un nouveau relai d̀ un régulateur par tout ou rien ordinaire permet de réduire considérablement le degré d'asservissement donc l'erreur « statique ».

2. Méthode d'étude simple des installations presque linéaires et munies de régulateurs contenant des relais par lout ou rien asservis ou non. Cette méthode est apparentée à celle dite des « séparatrices 》 voir [5] ef [7]) pour les installations linéaires.

3. Flude d'installations typiques munies de régulateurs par tout ou rien ordinaires.

4. La condition de stabilité peut être radicalement transformée par la présence d'imperfections même infiniment petites.
Brief study of some examples of installations having non-linear elements by way of illustrating the study method described in $n^{\text {os }} 3$ and 5 , 1952 of la Houille Blanehe. Comparison of the results with those obtained elsewhere. (in the few cases where, up to now, such results existed).

Several points are to be noted in particular. 1.- Study of an acceleration head, on-and-off governor of simple design: the addition of a further relay to an ordinary on-and-off governor makes it possible to reduce the degree of feedback, hence the "static" error, to a considerable extent.

2.-Simple study method for almost linear installations equipped with governors having on-and-off relays which are or are not fedback. This method is similar to that of the so-called "méthode des séparatrices (separators)" [5;7] which has been developed for linear installations.

3.-Sludy of typical installations equipped with ordinary, on-and-off governors.

4.- The stability condition may be radically altered by the presence of even infinitely small defects. 


\title{
TROISIE ME PARTIE*
}

\author{
SOMMAIRE
}

VI. - Exemples d'APplications de la méthode DE NyQUIST, QUELQUES PROPRIÉTÉS DES ÉLÉMENTS NON LINÉAIRES.

VI-1. - Cas élémentaires où les résultats sont en partie connus par ailleurs.

VI-11. - Régulateur direct de niveau avec jeu et adduction par conduite courte.

VI-12. - Régulateur par tout ou rien avec adduction par conduite unique et à coups de bélier en masse.
VI-2. - Cas solubles uniquement par la méthode de Nyqurst généralisée.

VI-21. - Régnlateur par tout ou rien et adduction par conduite unique à coups de bélier en onde.

VI-22. - Régulateur par tout ou rien et adduction comprenant une cheminée d'équilibre.

VI-23. - Régulateur à vitesse proportionnelle $\dot{a}$ l'écart et liaison à jeu.

VII. - Résumé final et conclusions.

\section{VI. - EXEMPLES D'APPLICATIONS DE LA METHODE DE NYQUIST. QUELQUES PROPRIETTÉS DES ÉLEMENTS NON LINEAARES.}

Nous avons exposé, dans les numéros 3 et $5 / 1952$ de la Houille Blanche la méthode que nous préconisons pour l'étude des installations à réglage automatique contenant des éléments à caractéristique non linéaire. Rappelons que jusqu'à présent il n'existait pas de méthode générale permettant d'étudier ces installations. Seules certaines installations très simples et très peu employées en pratique pouvaient être abordées. La méthode nouvelle préconisée ici permet d'étudier, plus ou moins rapidement et plus ou moins complètement, la plupart des installations que l'on rencontre en pratique. Nous allons l'employer ici à titre d'exemple pour analyser certaines propriétés de dispositifs non linéaires intervenant souvent dans les installations courantes. Comme on le sait, l'idéal en matière de régulation serait de trouver des formules littérales, traduisant les principales propriétés des diverses installations que l'on peut concevoir ou rencontrer. Malheureusement, les installations réelles sont souvent trop complicuées, et il serait illusoire de vouloir chercher des formules générales. Les problèmes qu'on peut résoudre en régulation sont de deux espèces :

D'abord on peut chercher à connaitre les principales propriétés des divers dispositifs susceptibles d'être employés; ce sont là des problèmes littéraux, mais plus simples que les études

\footnotetext{
* Cf. la Houille Blanche, $n^{\circ} 3-1952$, p. 394; n ${ }^{\circ}$ 5-1952, p. 665 .
}

globales d'installations réelles. Souvent ils consistent en l'étude de schémas suffisamment simples pour être facilement accessibles au calcul, mais suffisamment près de la réalité pour permettre de reconnaitre l'influence des divers paramètres et comparer divers dispositifs de conception voisine;

D'autre part on peut étudier des installations plus ou moins déterminées numériquement et chercher les «meilleures» valeurs à donner aux paramètres restant libres.

Nous avons montré, dans le numéro $5 / 1952$ de la Houille Blanche, page 681 , comment on pouvait étudier, au moyen de la méthode préconisée ici, une installation donnée numériquement. Dans la présente partie de notre étude, nous allons montrer comment cette méthode peut servir à étudier les propriétés générales de certains dispositifs non linéaires. En plus, nous comparerons les résultats obtenus par notre méthode aux résultats connus par ailleurs pour les quelques cas où il existe des résultats. On se rappelle en effet que la méthode proposée est une méthode approchée, dont nous avons néanmoins montré théoriquement que le degré d'approximation obtenu était très satisfaisant en pratique.

Nous allons étudier plus particulièrement les propriétés des régulateurs par tout ou rien, ef des transmissions ayant des imperfections équivalentes à un jeu. Pour étudier spécifiquement ces propriétés, nous simplifierons les installa- 
tions, bien plus qu'il ne serait nécessaire, pour permettre l'application de la méthode de Nyourst généralisée. En effet, même les calculs relativement simples exposés ici apportent des résultats nouveaux et intéressants. On pourrait graduellement augmenter les difficultés de l'étude en se rapprochant de plus en plus de la réalité et faire une étude beaucoup plus poussée de certaines catégories d'installations. Une telle étude (même littérale) serait possible, mais sortirait du cadre d'une simple présentation de méthode.

Dans les exemples traités ici, nous linéariserons tous les éléments, sauf les régulateurs à tout ou rien et les jeux. La linéarisation est pour les problèmes littéraux une simplification énorme; elle l'est beaucoup moins pour les problèmes numériques. Il en est de même des autres simplifications utilisées ici.

\section{VI-1. - CAS ELLEMENTAIRES OU LES RESULTATS SONT EN PARTIE CONNUS PAR AILLEURS}

\section{VI-11. - Régulateur direct de niveau avec jeu et adduction par conduite courte} (Voir fig. 1)

Cette installation peut comprendre ou non une conduite d'adduction. Nous étudierons le cas

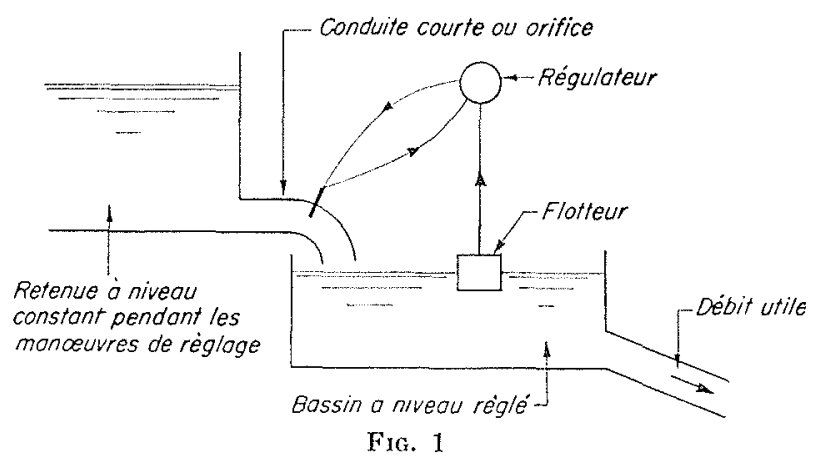

où l'inertie de l'eau dans l'adduction est négligeable. L'étude de problèmes analogues, où l'on a tenu compte de l'inertie de l'eau, montre que celle-ci est négligeable si le nombre $\frac{\mathrm{L}}{g_{\sigma}} \frac{\mathrm{Q}}{\mathrm{H}}$ est petit devant la base de temps de l'installation (cf. fréquence de coupure, § II-3) *.

Cette installation peut servir à «briser »la charge (elle diminue considérablement la charge dans le système à l'aval du bassin) ou à mainte-

\footnotetext{
* Les paragraphes I, II, III, IV ont été publiés dans la Houille Blanche, $n^{\circ}$ 3-1952. Le paragraphe $V$ dans la Houille Blanche, ${ }^{\circ}$ 5-1952.

S'il n'y a pas d'autres indications, les références se rapportent toujours à ces articles-là,
}

nir un niveau (ou un débit) constant. Le niveau dans la retenue peut varier beaucoup tout en restant constant pendant les manœuvres du régulateur. Par exemple : variations saisonnières.

Nous étudierons le cas d'une installation munie d'un régulateur direct, et nous admettrons que les imperfections de l'installation sont équivalentes à un jeu dans la transmission entre le niveau dans le bassin et l'entrée du régulateur. On voit facilement que, parmi ces imperfections, se classent par exemple d'autres jeux (entre le régulateur et la vanne, etc.) et certains frottements secs (ceux de la liaison entre le niveau et le régulateur, etc.).

Le phénomène de réglage est régi par les équations suivantes :

- Remplissage du bassin :

$$
\mathrm{S} \frac{d \mathrm{~N}_{1}}{d t}=q_{s}-q_{c}
$$

$S$ : surface du bassin.

$\mathrm{N}_{1}$ : niveau dans le bassin.

$q_{s}$ : débit sortant du bassin.

$q_{e}:$ débit entrant.

- Loi de l'orifice de remplissage :

$$
q_{e}=\mathrm{KY}
$$

Y : ouverture relative de la vanne.

$\mathrm{K}$ : coefficient de débit de la vanne.

- Loi de la transmission avec jeu :

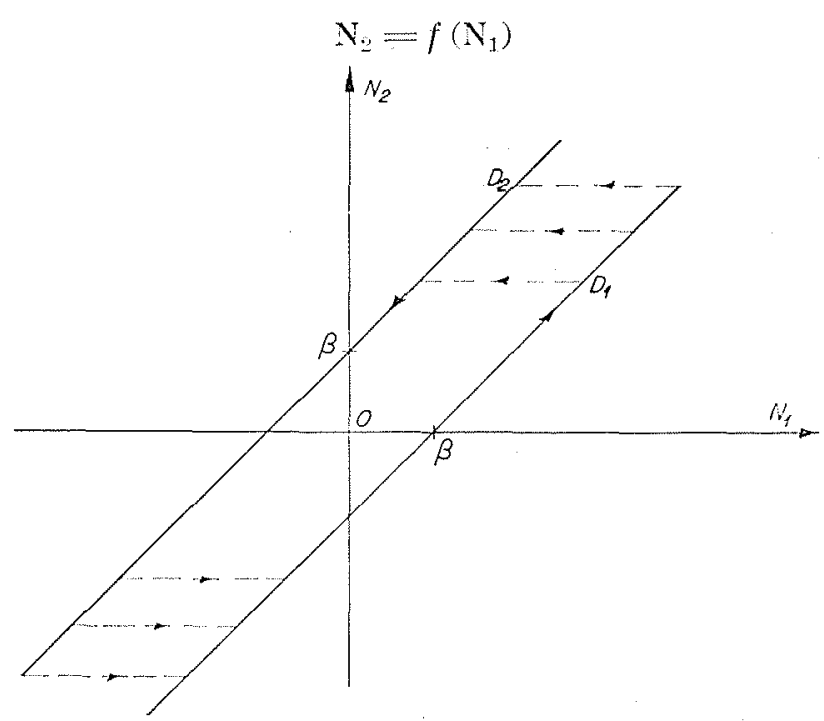

Fia. 2. - Si $\mathrm{N}_{1}$ croît, la liaison se fait selon $\mathrm{D}_{1}$; dans le cas contraire, selon $\mathrm{D}_{2}$. Aux changements de sens de variation de $N_{1}$, le point figuratif décrit une horizontale entre $\mathrm{D}_{x}$ et $\mathrm{D}_{2}$.

Cette loi est définie par la figure 2 où les droites $D_{1}$ et $D_{2}$ sont :

$$
\mathrm{N}_{2}=\mathrm{N}_{1} \pm \beta
$$


$\mathrm{N}_{2}$ : niveau tel que le « voit » le régulateur à travers la liaison:

$\beta:$ jeu *

(Voir aussi à ce sujet $\$$ V-232.)

- Loi de fonctionnement du régulateur:

Régulateur par tout ou rien :

$$
\frac{d \mathrm{Y}}{d t}=-\varepsilon \mathrm{V}_{0}
$$

s étant le signe de $\mathrm{N}_{2}-m \mathrm{Y}$.

$m$ : degré d'asservissement.

Régulateur à vitesse proportionnelle à l'écart:

$$
\frac{d \mathrm{Y}}{d t}=--\mathrm{K}_{0}\left(\mathrm{~N}_{2}-m \mathrm{Y}\right)
$$

Nous traiterons ici le cas du régulateur par tout ou rien qui est le « moins linéaire» (l'autre cas se traite de la même façon).

\section{a) Méthode directe:}

On élimine $d t$ entre :

$$
\frac{d \mathrm{Y}}{d l}=--s \mathrm{~V}_{0} \quad \text { et } \quad \mathrm{s} \frac{d \mathrm{~N}_{1}}{d t}=q_{e}-q_{\mathrm{s}}
$$

On étudie le phénomène de réglage dans les plans $\mathrm{N}_{1} ; \mathrm{Y}$ et $\mathrm{N}_{1} ; \mathrm{N}_{2}$.

On a :

$$
\mathrm{S} \frac{d \mathrm{~N}_{1}}{d \mathrm{Y}}=-\frac{q_{e}-q_{s}}{\varepsilon \mathrm{V}_{0}}
$$

Si $q_{s}$ est constant, on peut écrire :

$$
q_{e} \div q_{s}=\mathrm{KY}-q_{s}=\mathrm{K}\left(\mathrm{Y}-\mathrm{Y}_{0}\right)
$$

avec :

$$
\mathrm{Y}_{0}=\frac{q_{\mathrm{s}}}{\mathrm{K}}
$$

On en tire :

$$
\mathrm{N}_{1}-\mathrm{N}_{10}=-\varepsilon \frac{\mathrm{K}}{2 \mathrm{SV_{0 }}}\left(\mathrm{Y}-\mathrm{Y}_{0}\right)^{2}
$$

$\mathrm{N}_{10}$ étant une constante d'intégration.

Le phénomène est alor's le suivant : initialement, $\approx$ a un certain signe et le point figuratif décrit une parabole. Quand $\mathrm{N}_{2}-m \mathrm{Y}$, c'est-à-dire $N_{1} \pm \beta-m Y$ change de signe, $\varepsilon$ change aussi de

\footnotetext{
* Page 675, no 5-1952 de la Houllle Blanche. Nous
} avions posé : jeu $=2 \beta$. signe, et le point figuratif décrit une nouvelle parabole. L'équation de cette nouvelle parabole contient une nouvelle valeur $\mathrm{N}_{10}$ qui doit ètre définie de façon que la nouvelle parabole débute au point N.Y, auquel l'ancienne s'est arrêtée. La condition qui détermine $\mathrm{N}_{10}$ est linéaire el il n'y a pas d'ambiguité.

\section{Exemple pratique:}

$t<0 ; \quad q_{e}=q_{s} ; \quad \mathrm{Y}$ et $\mathrm{N}$ sont constants, $t=0 ; q_{s}$ varie brusquement de $q_{10}$ à $q_{0}$. $t>0 ; q_{s}=q_{0}$.

Dans ce cas, le phénomène de réglage est to suivant (voir fig. 3 et 4). A l'instant $t=0$, le point figuratif est sur le segment $A B$, e'est-à-dire que la valeur $\left(\mathrm{N}_{\Omega}-m \mathrm{Y}\right)$ est nulle et que $\mathrm{N}_{1}$ est compris entre $\mathrm{N}_{2}+\beta$ et $\mathrm{N}_{2}-\beta$ (dans la zone d'insensibilité du jeu). Admettons que le poinl figuratif soit en C. Puisque $q_{0}$ est inférieur à $\gamma_{10}, \mathrm{~N}_{1}$ commence à augmenter : le point figuratif se déplace de $\mathrm{C}$ en $\mathrm{B}$ à l'intérieur de la zone

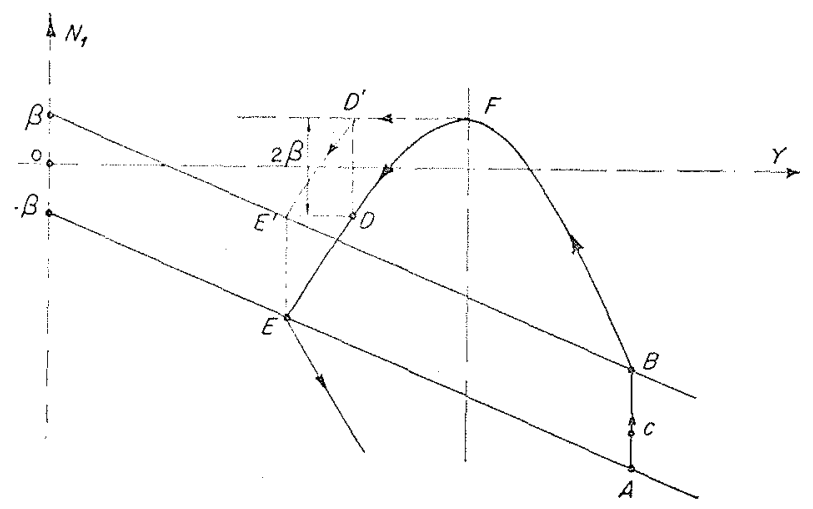

iric. 3

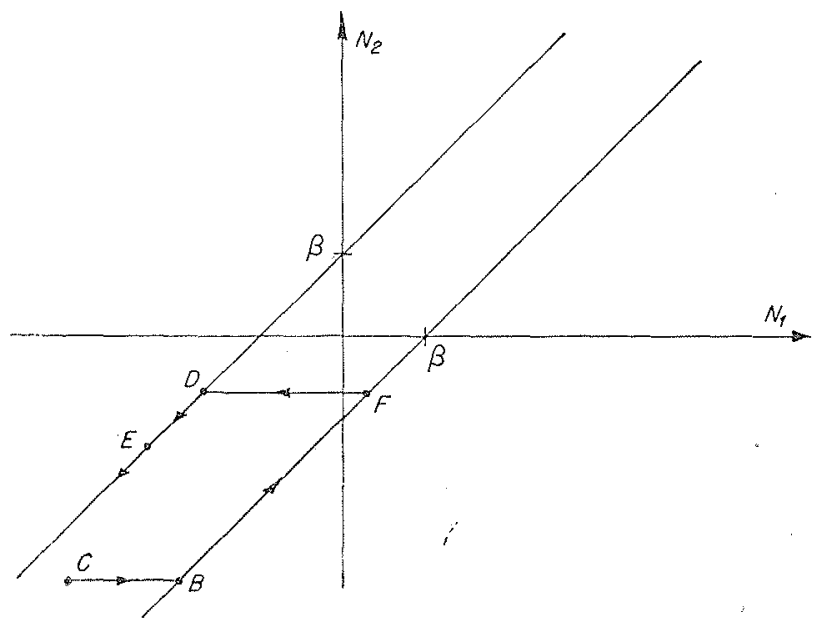

FIG, 4 
d'insensibilité. Le régulateur ne reçoit pas d'ordre et ne bouge pas.

En $B$, la quantité $N_{2}-m \mathrm{Y}$ devient positive et le régulateur commence à fonctionner. Le point figuratif décrit une parabole :

$$
\mathrm{N}_{1}-\mathrm{N}_{0}=-\frac{\mathrm{K}}{2 \mathrm{SV_{0 }}}\left(\mathrm{Y}-\mathrm{Y}_{0}\right)^{2}
$$

$\mathrm{N}_{0}$ étant à déterminer de façon que la parabole passe au point $\mathrm{B}$. Au point $\mathrm{F}, \mathrm{N}_{1}$ atteint un maximum, puis le point figuratif décrit FD. Dans le plan $\mathrm{N}_{1}, \mathrm{Y}$ nous avons également schématisé le trajet du point $N_{2}, Y$ en pointillé $\left(F \rightarrow D^{\prime}\right)$. A partir de $D$, le point figuratif décrit DE. En $E$, $\varepsilon$ change de signe, d'où une nouvelle parabole, dont la constante d'intégration est à déterminer par la condition exprimant que la nouvelle parabole passe au point $\mathrm{F}$. Et ainsi de suite.

Cherchons les cycles limites possibles.

Dans le plan $\mathrm{N}_{1}, \mathrm{Y}$, il ne peut exister que des cycles limites avant la forme représentée sur Ia figure 5. $P_{1}$ et $P_{2}$ sont deux paraboles symétriques. Le point $\mathrm{N}_{2}, \mathrm{Y}$ décrit le trajet en pointillé (analogue à celui de la figure 3 ). On voit que cette disposition n'est possible que si le point $B$. qui est sur la verticale de $M$, est à droite du point $A$, qui est sur l'horizontale de N. Dans le cas contraire, le mouvement a l'allure représentée sur les figures 6 et 7 . En $A^{\prime}$ on a $\mathrm{N}_{2}-m \mathrm{Y}=0$. On va montrer que le mouvement ultérieur se fait obligatoirement à $\mathrm{Y}$ constant. Comme le point figuratif dans le plan $\mathrm{N}_{1}, \mathrm{~N}_{3}$ est dans la zone d'insensibilité, $\mathrm{N}_{2}$ est constant. On en conclut que $\frac{d \mathrm{Y}}{d t}>0$ est incompatible avec $\mathrm{N}_{2}-m \mathrm{Y}<0$; de mème $\frac{d \mathrm{Y}}{d t}<0$ et $\mathrm{N}_{2}-m \mathrm{Y}>0$.

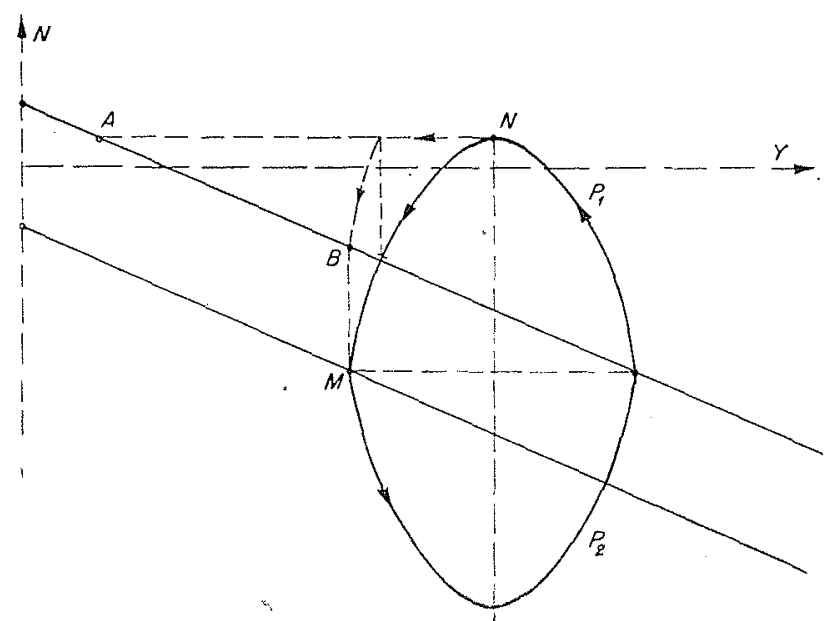

FIG. 5

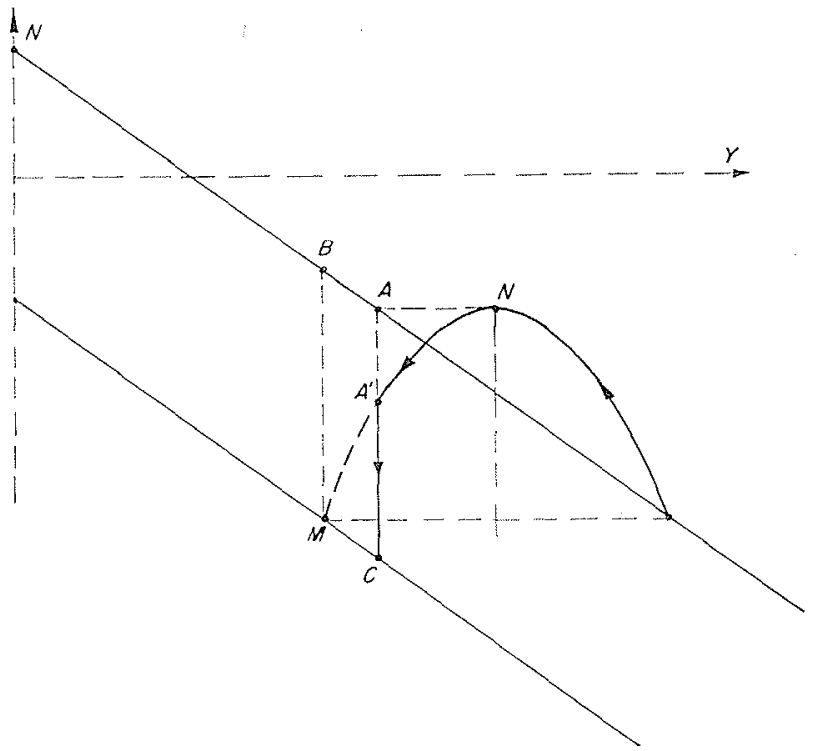

Fig. 6

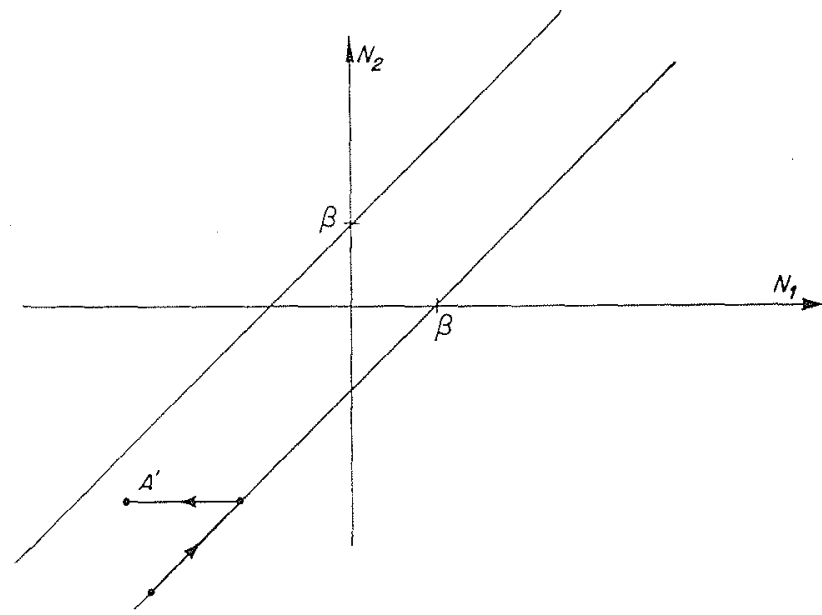

FIG. 7

Donc il faut que :

$$
\frac{d \mathrm{Y}}{d t}=0 \quad \text { et } \quad \mathrm{N}_{2}-m \mathrm{Y}=0
$$

ce qui est d'ailleurs compatible. $\mathrm{N}_{1}$ varie puisque $q_{s}-q_{e} \neq 0$. Donc le point figuratif décrit $\mathrm{A}^{\prime} \mathrm{C}$. On s'apercoit de proche en proche que le mouvement tend vers $\mathrm{Y}=\mathrm{Y}_{0}$; il ne peut pas $\mathrm{y}$ avoir de cycle limite mais il y a stabilité pure et simple. La limite entre les deux cas est obtenue quand les points $A$ et $B$ de la figure 5 sont confondus, on a alors :

$$
\beta=\frac{4 m^{2} V_{0} S}{K}
$$


Il y a cycle limite si :

$$
\beta>\frac{4 m^{2} V_{0} S}{K}
$$

Son amplitude est alors de $\frac{\beta}{m}$ en $Y$ et de $\frac{\mathrm{K} \beta^{2}}{2 \mathrm{~S} \mathrm{~V} \mathrm{~m}^{2}}$ en $\mathrm{N}$.

\section{b) Méthode par l'analyse harmonique:}

Rappelons qu'elle consiste (pour les régulateurs par tout ou rien) à déterminer le déphasage total que subit le fondamental d'une oscillation qui parcourt toute la boucle. Il y a cycle limite si ce déphasage est égal à $-\pi+2 k \pi$. La boucle est représentée sur la figure 8 .

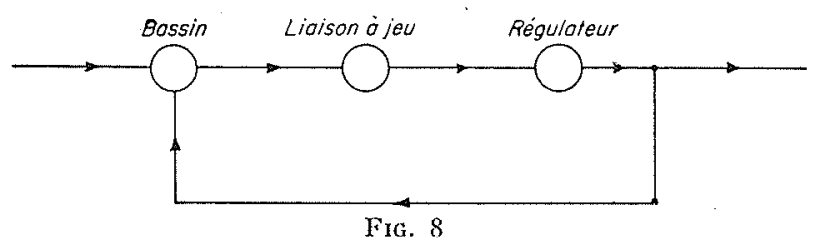

Pour rechercher ce déphasage, on peut admettre en première approximation qu'il est égal à la somme des déphasages de chaque élément auquel on injecte l'excitation A cos $\omega t$ (voir $\S$ V-232). En plus, on peut schématiser plus ou moins bien les éléments. En particulier une excitation A $\cos \omega t$ à l'entrée du régulateur donne à sa sortie un mouvement en «triangle» (voir fig. 9).

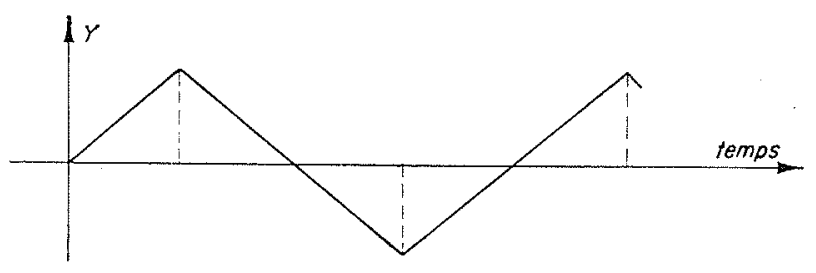

FIG. 9

Ce mouvement est réinjecté à l'entrée par l'asservissement. On peut le schématiser par son fondamental. De même, on peut schématiser plus ou moins bien le jeu. Toutes ces simplifications ne sont pas nécessaires pour l'application de la méthode, mais elles rendent les calculs littéraux plus aisés. Nous les ferons ici afin de montrer qu'avec un minimum de calcul, le phénomène est encore assez correctement représenté, même si on schématise très grossièrement l'installation. Dans ce cas on a les déphasages suivants :

\section{Régulateur :}

$$
\zeta=-\arccos \frac{4}{\pi} \frac{m V_{0}^{*}}{\omega\left|N_{2}\right|}
$$

(voir $\$$ V-232)

$$
\begin{array}{ll}
\text { Bassin : } & \varphi=-\pi / 2 \\
\text { Liaison }: & \varphi=-\arcsin \frac{\beta}{\left|\mathrm{N}_{1}\right|}
\end{array}
$$

(Voir \& V-232.)

D'où un cycle limite si :

$$
\frac{4}{\pi} \frac{m V_{0}}{\omega\left|N_{2}\right|}=\frac{\beta}{\left|N_{1}\right|}
$$

Le régulateur par tout ou rien impose a chaque fréquence une amplitude du cycle limite (comme on l'a déjà vu au $\S$ V-42). On peut done calculer les amplitudes de $N_{1}$ et $N_{2}$ en fonction de $\omega$; d'où trois équations dont on peut tirer les caractéristiques du cycle limite. Nous allons opérer un peu autrement en calculant simplement l'amplitude de $\mathrm{N}_{1}$ en fonction de $\omega$. On a successivement :

$$
\begin{aligned}
& |\mathrm{Y}|=\frac{4}{\pi} \frac{V_{0}}{\omega} \text { (voir \& V-232) } \\
& |\mathrm{Q}|=\mathrm{K}|\mathrm{Y}|=\mathrm{K} \frac{4}{\pi} \frac{\mathrm{V}_{0}}{\omega} \\
& \left|\mathrm{N}_{1}\right|=\frac{|\mathrm{Q}|}{\mathrm{S} \omega}=\frac{4 \mathrm{~V}_{0} \mathrm{~K}}{\pi} \mathrm{S \omega ^{2 }}
\end{aligned}
$$

D'où, par l'élimination de $\omega$ :

$$
\left(\frac{\left|\mathrm{N}_{1}\right|}{\beta}\right)^{3}=\frac{\pi}{4} \frac{\beta}{\mathrm{V}_{0} m^{2}} \frac{\mathrm{K}}{\mathrm{S}}\left(\frac{\left|\mathrm{N}_{2}\right|}{\beta}\right)^{2}
$$

D'autre part, la transmission avec jeu assure une relation entre $\left|N_{1}\right|$ et $\left|N_{2}\right|$ (voir $\S \mathrm{V}-232$ ).

$$
\frac{\left|N_{2}\right|}{\left|N_{1}\right|} \# 1-0,62 \frac{2 \beta}{\left|N_{1}\right|}-0,38\left(\frac{2 \beta}{\left|N_{1}\right|}\right)^{2}
$$

Il y a cycle limite possible si les deux relations entre $\frac{\left|N_{1}\right|}{\beta}$ et $\frac{\left|N_{2}\right|}{\beta}$ (1) et (2) admettent un groupe de racines réelles. Ces deux relations se traduisent par deux courbes dans le plan $\left|N_{1}\right| / \beta ;\left|N_{2}\right| / \beta$. L'allure de ces courbes est donnée par la figure 10. La courbe $\mathrm{C}_{1}$ est fixe, la courbe $C_{2}$ dépend d'un paramètre : $\frac{\beta}{V_{0} m^{2}} \frac{K}{S}$. Il y a un cycle limite possible si les deux courbes se coupent. On pourrait même croire qu'il y ait alors deux cycles limites, mais l'un provient de racines étrangères introduites par le calcul

\footnotetext{
* Les deux barres || signifient «amplitude de ».
} 
(voir à ce sujet une discussion faite à la fin du prochain paragraphe). La limite entre les cas où

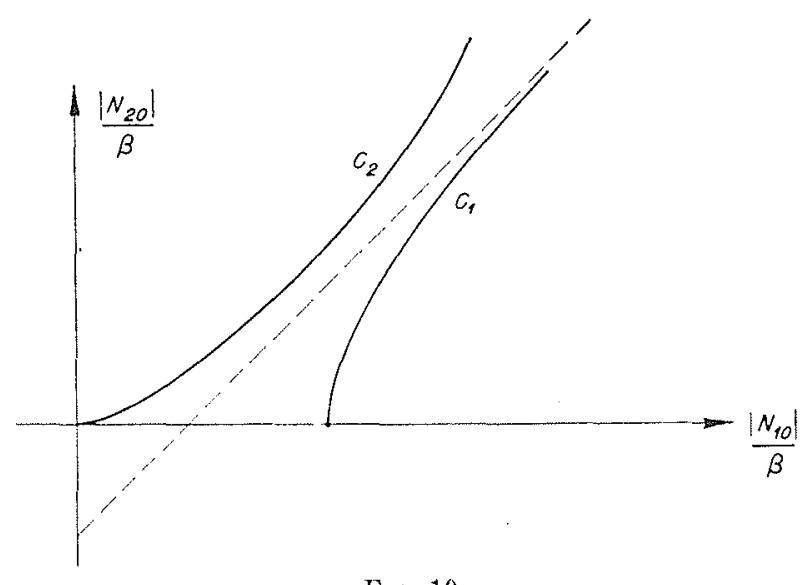

FIG. 10

il existe un cycle limite et les cas où il n'en existe pas est donnée par la condition de tangence des courbes $\mathrm{C}_{1}$ et $\mathrm{C}_{2}$. Cette condition est évidemment de la forme :

$$
\frac{\beta}{V_{0} m^{2}} \frac{\mathrm{K}}{\mathrm{S}}=\mathrm{C}^{\mathrm{ste}}
$$

Le calcul exact est faisable mais long. On obtient environ :

$$
\frac{\beta}{V_{0} m^{2}}-\frac{K}{S}=5,1 \ldots
$$

D’où :

$$
\beta=5,1 \ldots \frac{V_{0} m^{2} S}{K}
$$

Pour l'amplitude du cycle limite en $N_{1}$, on trouve :

$$
\left|\mathrm{N}_{1}\right| \# \frac{\pi}{4} \frac{\mathrm{K} \beta^{2}}{\mathrm{~S} V_{0} m^{2}}
$$

(la valeur réelle étant un peu plus petite).

On voit que, malgré les diverses approximations faites, les résultats ne sont pas loin de ceux trouvés par la méthode directe. En particulier, leur forme est bien la même (et celle-ci n'est pas la seule possible dimensionnellement). $\mathrm{Si}$, dans un cas particulier (numérique), on prend les fonctions de transfert plus exactes pour le jeu et aussi pour le régulateur, on trouve des résultats extrêmement voisins de ceux trouvés par la méthode directe.

\section{VI-12. - Régulateur par tout ou rien " avec adduction par conduite unique et à coups de bélier en masse (Fig. 11)}

L'installation est voisine de celle schématisée à la figure 1 ; néanmoins, ici, la conduite d'adduction est plus longue et l'inertie de l'eau dans celle-ci n'est plus négligeable. Bien que cette installation soit très simple, les méthodes habituelles ne permettent plus de l'étudier. Cependant, on connaît la condition de stabilité par expérience dans le cas où les pertes de charge dans la conduite sont négligeables. On sait que la condition de stabilité est alors la mème que celle d'une installation identique linéarisée et équipéc avec un certain régulateur à vitesse proportionnelle à l'écart. Ce régulateur doit établir la relation :

$$
\frac{d \mathrm{Y}}{d t}=-\mathrm{K}_{0}(\mathrm{~N}-m \mathrm{Y})
$$

où $\mathbf{K}_{0}$ tend convenablement vers 0 (ce point sera précisé par la suite).

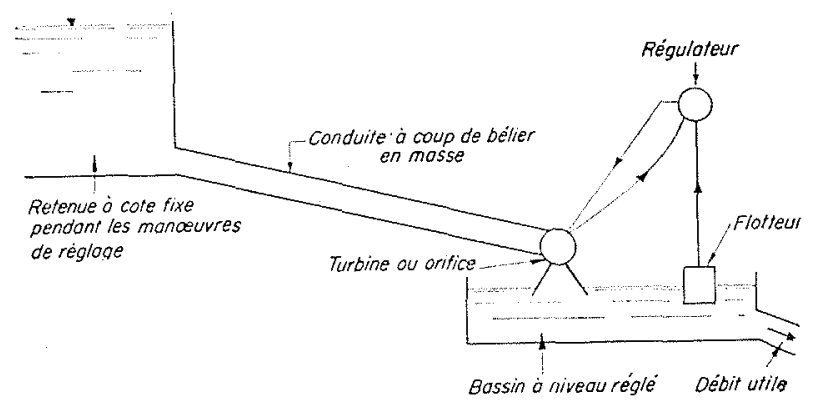

FIG. 11

Etudions d'abord l'installation avec le régulateur par tout ou rien. Les équations du phénomène sont dans ce cas :

-...- Remplissage du bassin (mèmes notations qu'au $\$$ VI-11).

$$
\mathrm{S} \frac{d \mathrm{~N}}{d t}=q_{s}-q_{e}
$$

- Coup de bélier dans l'adduction.

$$
\mathrm{H}_{s}-\frac{\mathrm{L}}{g_{0}} \frac{d q_{c}}{d t}=\mathrm{K}^{\prime} \frac{q_{e}^{2}}{\mathrm{Y}^{2}}
$$

$\mathrm{H}_{s}$ : charge statique.

L : longueur de la conduite.'

* Les régulateurs par tout óu rien asservis ne sont pas des éléments « algébriques $»$ (voir $\S$ V-234) comme on pourrait le croire a priori. Ils ne peuvent donc pas être étudiés par la méthode de MM. Dưruh et RochenuUnier ([3] et [4]). 
$\sigma$ : section de la conduite.

$\mathrm{K}^{\prime}$ : coefficient de perte de charge de l'orifice.

Régulateur : $\quad \frac{d Y}{d t}=--\varepsilon \mathrm{V}_{0}$

a étant le signe de $\mathrm{N}-m \mathrm{Y}$.

Pour les raisons exposées plus haut, nous linéariserons l'équation du coup de bélier dans la conduite. On peut d'ailleurs remarquer en passant que cette dernière non-linéarité est du type « linéarisable » (voir $\S \mathrm{V}-41$ ), tandis que l'équation du régulateur est du type « non linéarisable $»$. On peut dire que cette dernière non-linéarité est beaucoup plus «importante» que l'autre.

Nous posons done :

$$
\begin{aligned}
& q_{e}-q_{s}=\Delta q \\
& \mathrm{~N}-\mathrm{N}_{0}=\Delta \mathrm{N} \\
& \mathrm{Y}-\mathrm{Y}_{0}=y
\end{aligned}
$$

$\mathrm{N}_{0}$ et $\mathrm{Y}_{0}$ étant les valeurs de $\mathrm{N}$ et $\mathrm{Y}$ en régime permanent si le débit est $q_{*}$.

Près de ce point de fonctionnement on a les fonctions de transfert suivantes:

Pour le bassin :

$$
\mathrm{S} j \omega \Delta \mathrm{N}=\Delta q
$$

Pour la conduite d'adduction :

$$
\Theta j \omega \frac{\Delta q}{q_{s}}+\frac{2 \Delta q}{q_{s}}=\frac{2 y}{\mathrm{Y}_{0}}
$$

dans laquelle :

$$
\Theta=\frac{\mathrm{L}}{g \sigma} \frac{q_{s}}{\mathbf{H}_{s}}
$$

Les déphasages sont donc:

Pour le bassin : $\quad-\pi / 2$

Pour la conduite : $\quad--\operatorname{arctg} \frac{\Theta \omega}{2}$

Pour le régulateur : $-\arccos \frac{4}{\pi} \frac{m V_{0}}{\omega|\Delta N|}$

On obtient la condition de cycle limite comme précédemment :

$\ldots \pi / 2-\operatorname{arctg} \frac{\Theta \omega}{2}-\arccos \frac{4}{\pi} \frac{m \mid \Delta \mathrm{V}_{0}}{\omega|\Delta \mathrm{N}|}=-\pi+2 k \pi$

D'où :

$$
\frac{4}{\pi} \frac{m V_{0}}{\omega|\Delta N|}=\frac{2}{\sqrt{4+\Theta^{2} \omega^{2}}}
$$

Comme précédemment, on peut chercher l'amplitude du cycle limite en $\mathrm{N}$ en fonction de $\omega$. On obtient successivement les amplitudes suivantes:

Le régulateur donne:

$$
|\mathrm{Y}|=\frac{4}{\pi} \frac{\mathrm{V}_{0}}{\omega}
$$

Lil conduite :

$$
|\Delta q|=\frac{2}{\sqrt{4+\Theta^{2} \omega^{2}}} \frac{q_{s}}{\mathrm{Y}_{0}}|\mathrm{Y}|
$$

Le bassin :

$$
|\Delta \mathrm{N}|=\frac{1}{\mathrm{~S} \omega}|\Delta q|
$$

d'où :

$$
|\Delta N|=\frac{4}{\pi} \frac{V_{0}}{\omega} \frac{2}{\sqrt{4+\Theta^{2} \omega^{2}}} \frac{q_{s}}{\mathrm{Y}_{0}} \frac{1}{\mathrm{~S} \omega}
$$

En éliminant $|\Delta N|$ entre les relations (5) et (6), on obtient l'équation donnant les pulsations des cycles limites possibles :

$$
\Theta^{2} \omega^{2}=\frac{2 \Theta q_{s}}{S m \mathrm{Y}_{0}}-4
$$

Pour qu'il y ait un cycle limite possible, il faut que les racines en $\omega$ de cette équation soient réelles, done :

$$
\Theta q_{s}>2 m \mathrm{SY}_{0}
$$

Si $\Theta q_{s}<2 S m Y_{0}$, on se rend compte que la somme des déphasages successifs n'atteint jamais $-\pi$; l'installation est alors absolument stable.

Si on remplace le régulateur par tout on rien par le régulateur à vitesse proportionnelle à l'écart défini plus haut, il faut joindre aux équations (3) et (4) l'équation du nouveau régulateur :

$$
\frac{d y}{d t}=-\mathrm{K}_{0}(\Delta \mathrm{N}-m y)
$$

où $\mathrm{K}_{0}$ tend convenablement vers zéro.

L'équation caractéristique est alors :

$\Theta p^{3}+p^{2}\left(2+\mathrm{K}_{0} m \Theta\right)+2 \mathrm{~K}_{0} m p+\frac{2 q_{s} \mathrm{~K}_{0}}{\mathrm{~S} \mathrm{Y}}=0$ et la condition de stabilité :

$$
2 \mathrm{SY} m\left(1+\frac{\mathrm{K}_{0} m \Theta}{2}\right)>q_{s} \Theta
$$


Si on fait tendre $K_{0} m \Theta \rightarrow 0$, on obtient :

$$
2 \mathrm{SY} m>q_{s} \Theta
$$

ce qui est la même condition que celle trouvée précédemment. Ce fait est bien connu depuis longtemps. Il a donné lieu à divers commentaires. Parfois on prétendait que le régulateur par tout ou rien régle comme un régulateur infiniment lent. Souvent on prétendait aussi qu'on peut remplacer pour tous les calculs de stabilité le régulateur par tout ou rien par un régulateur à vitesse proportionnelle à l'écart infiniment lent. Ces deux assertions ne sont pas exactes. D'abord on ne peut pas conclure de la condition de stabilité que le régulateur par tout ou rien règle comme un régulateur infiniment lent. En effet, la rapidité de réglage n'est pas directement liée à la condition de stabilité. D'autre part, cette conclusion est contredite par l'étude de certaines installations, par exemple de celles où l'inertie de l'eau dans la conduite d'adduction est négligeable, et aussi par toutes sortes d'autres exemples qu'on peut traiter avec la méthode préconisée ici, en appliquant les théories du \$ V-41. On peut plutôt dire qu'un régulateur par tout ou rien donne un fonctionnement aussi stable qu'un l'égulateur à vitesse proportionnelle à l'écart manœuvrant beaucoup plus lentement. Par ailleurs, on montrera que l'installation en questioni est la seule pour laquelle le régulateur par tout ou rien est équivalent à un régulateur à vitesse proportionnelle à l'écart, en ce qui concerne la condition de stabilité.

Nous allons, dés à présent, «expliquer» en partie pourquoi cette équivalence est valable dans le cas traité ici : à sa limite d'existence le cycle limite a une amplitude infinie. Le régulateur à vitesse proportionnelle à l'écart, qui donne une vitesse de vannage finie pour une amplitude d'entrée infinie, doit avoir une rapidité de réponse qui tend convenablement vers zéro (voir fig. 12).

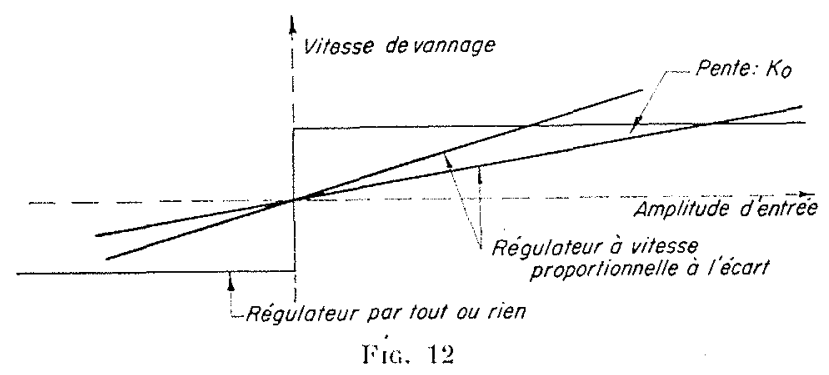

D'une façon tout à fait analogue on peut montrer que le régulateur par tout ou rien accélérotachymétrique n'est équivalent à un régulateur accélérotachymétrique à vitesse proportionnelle à l'écart que dans le cas de l'installation schématisée sur la figure 11.

On peut concevoir différents types de régulateurs accélérotachymétriques par tout ou rien. Nous allons décrire les deux principaux :

Le premier comprend deux relais dont l'un détecte les changements de signe de $\mathrm{N}$ et l'autre les changements de signe de $d N / d t$, e'està-dire pratiquement les maxima et minima de $\mathrm{N}$. Le moteur est soumis à une tension $\approx \mathrm{U}_{1}+\varepsilon^{\prime} \mathrm{U}_{2}$ et tourne à la vitesse $\varepsilon V_{1}+\varepsilon^{\prime} V_{2}{ }^{*}$.

Le deuxième comprend un seul relai qui est actionné par une tension proportionnelle à $\mathrm{N}+k d \mathrm{~N} / d t$ ( $\mathrm{N}$ étant le niveau détecté). On voit que pour le premier type le déphasage entre $\mathrm{N}$ et $\mathrm{Y}$ est $-\pi / 2+\operatorname{arctg}\left(\mathrm{V}_{2} / \mathrm{V}_{1}\right)$; pour le deuxieme il est $-\pi / 2+$ are tg $k \omega$. Si on règle une instalIation du genre de celle schématisée sur la figure 11 par un régulateur du premier type d'installation, il existe toujours un cycle limite. La pulsation correspondante est donnée par :

$\pi+2 k \pi=-\pi / 2-\pi / 2+\operatorname{arctg} \frac{V_{2}}{V_{1}}-\operatorname{arctg} \frac{\Theta \omega}{2}$

d'où :

$$
\omega=\frac{2 V_{2}}{V_{1}} \frac{1}{\Theta} \text { * * }
$$

Avec le deuxieme type de régulateur il y a stabilité ou instabilité suivant que:

$-\pi / 2-\pi / 2+\operatorname{arctg} k \omega-\operatorname{arctg} \frac{\Theta \omega}{2} \geqslant-\pi$

Done :

$$
k \equiv \frac{\Theta}{2}
$$

On voit que ce dernier type de régulateur est le plus stable. Pour étudier la stabilité de l'installation munie d'un régulateur accélérotachymétrique à vitesse proportionnelle à l'écart, il faut joindre aux équations la relation établie par ce régulateur, soit :

$$
\frac{d y}{d t}=-\mathrm{K}_{0} \mathrm{~N}-\mathrm{K}_{1} \frac{d \mathrm{~N}}{d t}
$$

l'équation caractéristique est :

$$
\Theta p^{3}+2 p^{2}+\frac{2 \mathrm{~K}_{1} q_{s}}{\mathrm{SY_{0 }}} p+\frac{2 \mathrm{~K}_{0} q_{s}}{\mathrm{~S}_{0}}=0
$$

d'où la condition de stabilité :

$$
\frac{\mathrm{K}_{1}}{\mathrm{~K}_{0}}>\frac{\theta}{2}
$$

* Pratiquement on peut d'ailleurs réaliser deux vitesses de vannage différentes avec un moteur tournant toujours à la même vitesse et un réducteur.

** $\mathrm{Si} \mathrm{Y}_{2} / \mathrm{V}_{\mathrm{I}}$ est négatif, i] $\mathrm{y}$ a instabilité apériodique. 
Cette condition est la mème que (7).

Des deux types de régulateur par tout ou rien mentionnés ici, l'un domne un fonctionnement moins stable; mais il est beaucoup plus simple à réaliser puisqu'on ne mesure aucune grandeur; on détecte simplement des zéros, des maxima et des minima. Ce dernier régulateur peut être stabilisé par un asservissement beaucoup plus faible que celui exigé par un régulateur seulement tachymétrique (facilement de l'ordre de 1 à 50 en pratique). Ce régulateur est donc robuste, simple et bien stable.

Etudions l'installation schématisée par la figure 11 réglée par un tel régulateur.

Soit $\mathrm{N}=\mathrm{N}_{0} \cos \omega t$ le mouvement du niveau dans le bassin et $\mathrm{Y}=\mathrm{Y}_{0} \cos (\omega t-p)$ le mouvement de la vanne.

On sait que :

$$
\pi / 2 \geqslant \varphi \geqslant 0
$$

L'un des relais recoit la tension :

$$
\begin{aligned}
\mathrm{N}-m \mathrm{Y}=\mathrm{N}_{0} \cos \omega t-m \mathrm{Y}_{0} \cos (\omega t-\varphi) \\
=\mathrm{A} \cos (\omega t+\downarrow)
\end{aligned}
$$

On sait que :

$$
\pi / 2 \geqslant \psi \geqslant 0
$$

On en déduit la relation :

$$
\mathrm{N}_{0} \sin \psi=m \mathrm{Y}_{0} \sin (\varphi+\psi)
$$

L'autre relai reçoit la tension :

$$
\frac{d \mathrm{~N}}{d t}=-\mathrm{N}_{0} \omega \sin \omega t
$$

Le régulateur donne une vitesse de vannage :

$$
\frac{d Y}{d t}=-\frac{4}{\pi} V_{0} \cos (\omega t+\psi)+\frac{4}{\pi} V_{1} \sin \omega t
$$

avec la même approximation qu'aux $\S \S$ VI-11 et VI-12.

D'où :

$Y_{0} \cos (\omega t+\varphi)=-\frac{4}{\pi} \frac{V_{0}}{\omega} \sin (\omega t+\psi)-\frac{4}{\pi}-\frac{V_{1}}{\omega} \cos \omega t$

On en déduit :

$$
\begin{aligned}
& \mathrm{Y}_{0} \sin \varphi=-\frac{4}{\pi} \mathrm{V}_{0} \cos \psi \\
& \mathrm{Y}_{0} \cos \varphi=-\frac{4}{\pi} \mathrm{V}_{0} \sin \psi-\frac{4}{\pi} \frac{\mathrm{V}_{1}}{\omega}
\end{aligned}
$$

L'étude du régulateur en soi peut être faite à partir de ces relations. Elle est assez longue et compliquée. Etudions ici directement les cycles limites qui peuvent se produire. La condition du cycle limitè est comme précédemment :

$$
-\pi / 2-\varphi-\operatorname{arctg} \frac{\Theta \omega}{2}=-\pi
$$

D'où :

$$
\operatorname{ctg} \varphi=\frac{\Theta \omega}{2}
$$

Pour étudier les cycles limites, il faut $\mathrm{y}$ joindre, comme au $\S$ VI-11, la relation entre $N_{0}$, $\mathrm{Y}_{0}$ et $\omega$. Elle est ici :

$$
N_{0}=\frac{2 Y_{0} Q}{Y}-\frac{1}{\sqrt{4+\Theta^{2} \omega^{2}}} \frac{1}{S \omega}
$$

Pour trouver l'équation en $\omega$ des cycles limites, nous éliminerons d'abord $Y_{0}$ entre les équations (9) et (10) et $N_{0}$ entre (8) et (12). D'où les relations :

$$
\cos (\varphi+\psi)=\frac{V_{1}}{V_{0}} \sin \varphi
$$

et :

$\frac{2 \mathrm{Q} \Theta}{m \mathrm{SY}} \frac{1}{\Theta \omega} \frac{1}{\sqrt{4+\Theta^{2} \omega^{2}}} \sin \psi=\sin (\varphi+\psi)(1$

on pose :

$$
\frac{2 \mathrm{Q} \Theta}{m \mathrm{SY}}=-a
$$

et on calcule $\sin \varphi$ et $\cos \varphi$ de l'équation (11). Puis on remplace dans (13) et (14) sin 9 et cos? par leur valeur. On obtient les deux relations :

$$
2 \cos \psi=\left(\frac{a}{\Theta \omega}-\Theta \omega\right) \sin \psi
$$

et :

$$
\Theta \omega \cos \psi-2 \sin \psi=2 \frac{V_{1}}{V_{0}}
$$

On peut en tirer :

$$
\begin{gathered}
\sin \psi \text { et } \cos \psi \\
\sin \psi=2 \frac{V_{1}}{V_{0}} \frac{1}{\frac{a}{2}-2-\frac{\Theta^{2} \omega^{2}}{2}} \\
\cos \psi=2 \frac{V_{1}}{V_{0}} \frac{\frac{1}{2}\left(\frac{a}{\Theta}-\Theta \omega\right)}{2-2-\frac{\Theta^{2} \omega^{2}}{2}}
\end{gathered}
$$

Jusqu'ici, nous n'avons pas introduit de raci- 
nes étrangères, puisque tous les calculs étaient linéaires. Pour éliminer $\psi$, il nous faudra écrire :

$$
\sin ^{2} \psi+\cos ^{2} \psi=1
$$

d'où I'équation des $\omega$ des cycles limites. Néanmoins ici on introduit des racines étrangères qu'on peut éliminer en recalculant $\sin \psi$ et $\cos \psi$ à partir des valeurs trouvées. Comme $\psi$ doit être compris entre 0 et $\pi / 2$, il suffit de vérifier que $\sin \psi$ et $\cos \psi$ sont positifs. L'équation en $\omega$ s'écrit (on pose $a-4=c$ et $\frac{4 \mathrm{~V}_{1}^{2}}{\mathrm{~V}_{0}}=b$ ) :

$$
\begin{aligned}
& f\left(\Theta^{2} \omega^{2}\right)=\left(\Theta^{2} \omega^{2}\right)^{3}-\left(\Theta^{2} \omega^{2}\right)^{2}(2 c+b) \\
& +(\Theta \omega)\left(c^{2}+2 b c+4 b\right)-b(c+4)^{2}=0
\end{aligned}
$$

Pour que $\sin \psi$ et $\cos \psi$ soient positifs, il faut et suffit que $c$ soit plus grand que la racine $\Theta^{2} \omega^{2}$ adoptée. Or, $f(c)<0$, donc il ne peut $\mathrm{y}$ avoir de cycle limite si $f\left(\Theta^{2}\left(\omega^{2}\right)=0\right.$ n'a qu'une racine réelle (fig. 13).

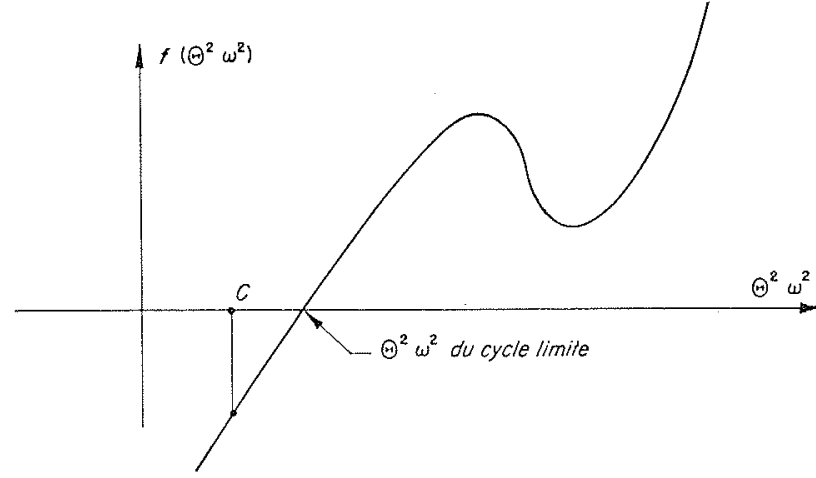

Fig. 13

Si $f\left(\Theta^{2} \omega^{2}\right)=0$ a 3 racines réelles, il faut que $c$ soit inférieur aux racines de $f^{\prime}\left(\theta^{2} \omega^{2}\right)=0$. pour ne pas avoir de cycle limite (fig. 14).

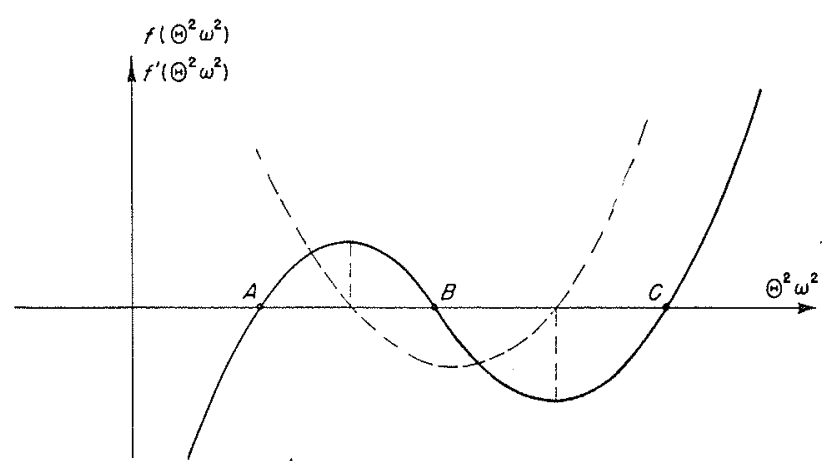

FIG. 14. - Puisque $f(c)<0, c$ peut être entre B et $\mathrm{C}$ ou à gauche de $A$. Pour que $c$ soit à gauche de $A$, il faut que $f^{\prime}(c)>0$, et $c$ à gauche du minimum de $f^{\prime \prime}(c)$ (voir fig. 15).

Or :

$$
f^{\prime}(c)>0
$$

Il faut done calculer la racine de $\left.\left.f^{\prime \prime}((-))^{\prime \prime(1)}\right)^{2}\right)=0$ el la comparer avec $c$ (fig. 15).

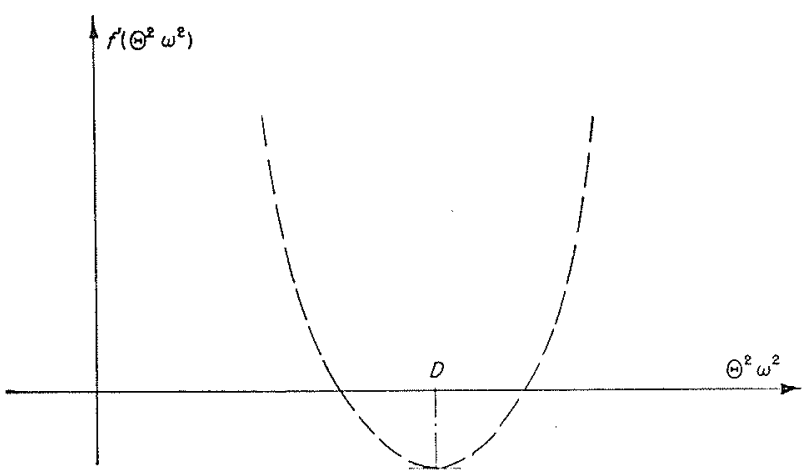

Fic. 15. - Puisque $f^{\prime}(c)>0$, il faut que $c$ soit à gauche de $D$ pour qu'il n'y ait pas de cycle limite.

On trouve facilement qu'il peut y avoir un cycle limite uniquement dans le cas où $f\left(\Theta^{2} \omega^{2}\right)=0$ a trois racines réelles et que $c<b$.

La condition de racine double qui limite le domaine où $f\left(\Theta^{2} \omega^{2}\right)=0$ a trois racines réelles, se trouve facilement en changeant de variables; on pose :

$$
\begin{gathered}
y=\Theta^{2} \omega^{2}-c \\
y^{3}+y^{2}(c-b)+4 b y-4 b(c+4)=0
\end{gathered}
$$

Les conditions de racine double sont :

$$
\begin{aligned}
& b[9(c+4)+(c-b)]^{2} \\
& =\left[12 b-(c-b)^{2}\right][3(c-b)(c+4)-4 b]
\end{aligned}
$$

ct $b=0$

La première représente une courbe $\mathrm{C}$ du $4^{\circ}$ degré en $b$ et $c$; cette courbe a trois points doubles en $b=4 ; c=4$ et $c=\frac{-88 \pm j 4 \sqrt{3}}{27}$ (voir $\S$ VI-2). Elle est donc unicursale. Elle n'est pas coupée par $c=0 ; b>0$, ni par $c=b ; b>0$. Elle ne peut entrer dans le domaine défini par $b=c$ et $b=0$ que par l'origine. A l'infini elle présente une branche parabolique du $3^{\circ}$ degré

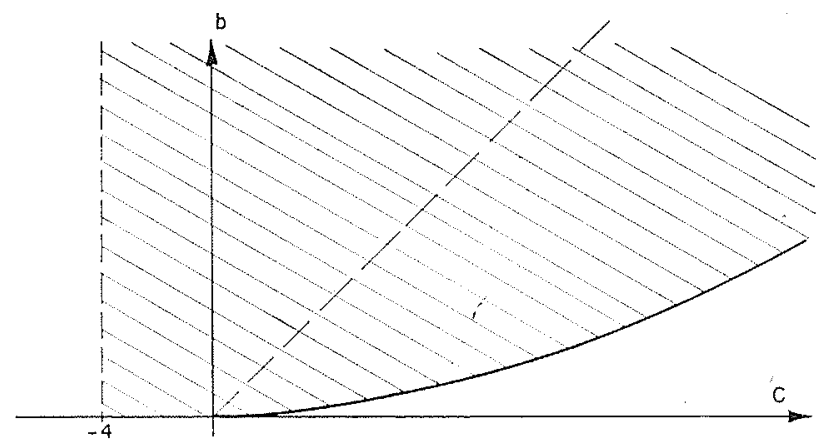

FIG. 16 
dans la direction $b=c$ et une asymptote dans la direction $c=0$. On en déduit que la forme de la courbe $\mathrm{C}$ est obligatoirement celle donnéc par la figure 16. La partie hachurée est la partie stable sans cycle limite.

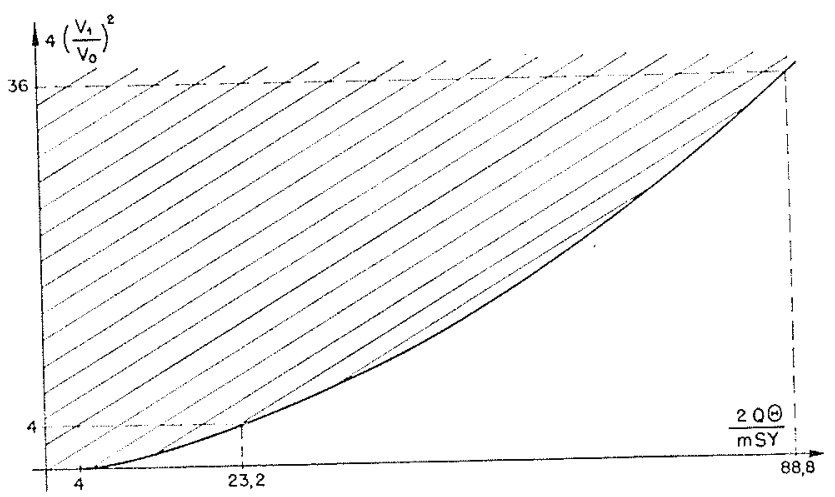

Fiti. 17. - Domaine de stabilité - hachure - (sans cycle limite) dans le plan:

$2 Q \Theta / m S Y, 4\left(V_{1} / V_{0}\right)^{2}$

La figure 17 montre le domaine de stabilité dans le plan $a=\frac{2 \mathrm{Q} \Theta}{m \mathrm{SY}}$ et $b=4\left(\frac{\mathrm{V}_{1}}{\mathrm{~V}_{0}}\right)^{2}$

Si $V_{1}=V_{0}$, on trouve la limite de stabilité (sur la courbe C) $a=12+8 \sqrt{2} \# 23,2$; le degré d'asservissement limite est déjà divisé environ par 6. On n'a pas intérêt à se placer vers $V_{1}=V_{0}$ : l'amortissement $y$ est asse $z$ mauvais, comme on le verrait par une étude plus poussée.

Les vitesses auxquelles doit tourner l'arbre de

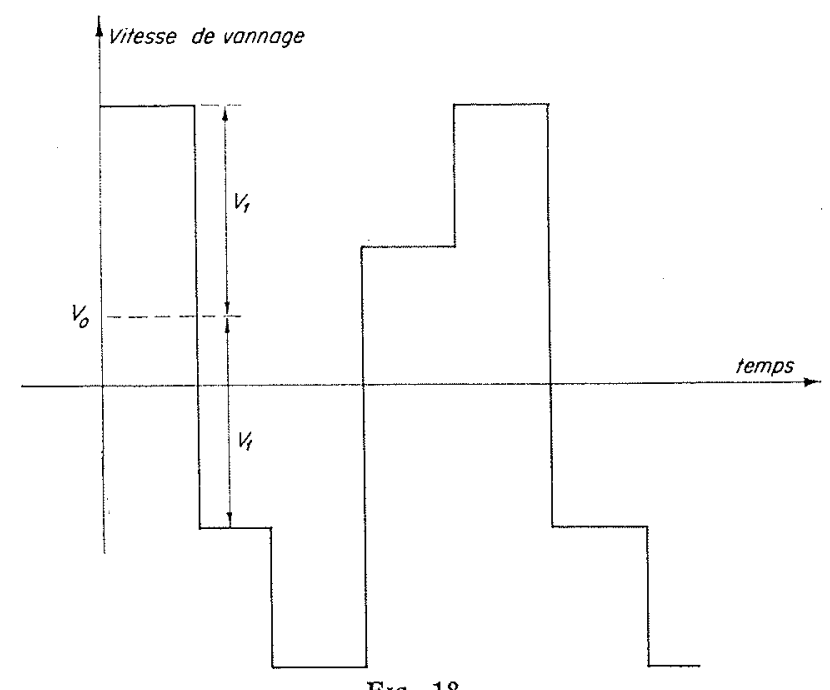

Fxa. 18

sortie $\mathrm{du}$ moteur du régulateur sont $\mathrm{V}_{0}+\mathrm{V}_{1}$ et $\mathrm{V}_{0}-\mathrm{V}_{1}$ (fig. 18). Si $\mathrm{V}_{1}$ est plus grand que $\mathrm{V}_{0}$, l'une des vitesses est en sens inverse de l'autre. La stabilité augmente, comme on le voit sur la figure 17 , si les vitesses de vannage tendent à être égales et de signe contraire. Une étude plus poussée montrerait que l'amortissement optimum correspond sensiblement à la valeur $1 / 2$ du rapport des deux vitesses. Dans ce cas, $\mathrm{V}_{0}=\mathrm{V}_{1} / 3$, donc $b=36$. Alors, la valeur du paramètre $a$ donnant la limite d'existence d'un cycle limite est environ 88,8 . On gagne donc déjà de 1 à 22 par rapport au régulateur par tout ou rien ordinaire. On peut facilement pousser la limite de stabilité plus loin encore en faisant tendre le rapport des vitesses de vannage vers - 1 , mais l'amortissement redevient alors de plus en plus faible.

Par ailleurs, on peut également employer ce régulateur sans asservissement. Comme on l'a déjà vu, il existe toujours un cycle limite. Néanmoins, celui-ci pent être très petit. Ainsi par exemple, si on admet une surpression de $20 \%$ dans la conduite d'adduction, et si $V_{0}=V_{1} / 3$, l'amplitude du cycle limite est environ 800 fois plus petite que la différence des niveaux extrêmes réglés par suite de l'asservissement nécessaire à la stabilité avec un régulateur par tout ou rien à une seule vitesse du vannage. Souvent le pompape a tendance à se produire à une fréquence supérieure à la fréquence de coupure (voir $\S$ II-3) et, de ce fait, peut ne pas apparaitre.

\section{VI-2. - CAS SOLUBLES UNIQUEMENT PAR LA METHODE DE NYQUIST GENÉRALISÉE}

Comme nous l'avons indiqué au début du $\$$ VI-1, nous nous proposons d'étudier surtout les régulateurs par tout ou rien et les liaisons à jeu. Pour étudier d'une façon assez générale ces deux éléments dans une installation par ailleurs linéarisée (comme nous l'avons indiqué plus haut), il est commode de chercher d'une façon systématique les fonctions de transfert des parties linéaires de ces installations. Les installations étudiées ici seront des installations hydrauliques et la partie la plus délicate de la fonction de transfert est celle provenant de l'adduction. Pour caractériser l'adduction, nous définirons une fonction que nous appellerons « l'impédance de l'adduction ${ }^{*}$. Cette fonction caractérise entièrement l'adduction dans le domaine des équations linéarisées. Nous la définirons de la façon suivante: on considère le système d'adduction muni de son orifice, mais sans régulateur. Si on impose à cet orifice une loi de manœurre de façon

* Cette notion se trouve plus largement développée dans l'un de nos récents articles : «Aptitude des installations hydrauliques à être réglées automatiquement $\gg$, Revue Générale de l'Electricité; janv. 1953 ; p. 40. 
telle que le débit soit $q(t)$, il se crée dans tout le système d'adduction des surpressions dues à l'inertie de l'eau. Soit $h(t)$ la surpression juste à l'amont de l'orifice. Comme nous nous plaçons dans le domaine des équations linéarisées et que nous étudions les régimes sinusoïdaux, il correspond à chaque fonction

$$
q(t)=\mathrm{Q}_{0} \cos \omega t=\mathcal{R}\left(\mathrm{Q}_{0} e^{j \omega t}\right)^{\star} .
$$

Une fonction :

$$
\begin{aligned}
h(t) & =\Re\left[Q_{0} Z(j \omega) e^{j \omega t}\right] \\
& =\mathcal{R}\left[H_{0}(j \omega) e^{j \omega t}\right]
\end{aligned}
$$

où $\mathrm{H}_{0} e^{j \varphi}$ est une fonction de $j \omega$, qui est de la forme :

$$
Q_{0} Z(j \omega)
$$

Done à :

$$
q(t)=\mathcal{R}\left(Q_{0} e^{e^{j \omega t}}\right)
$$

correspond :

$$
h(t)=\Omega\left[Q_{0} Z(j \omega) e^{j \omega t}\right]
$$

où :

$$
\mathrm{H}_{0}(j \omega)=Q_{0}(j \omega) \cdot Z(j \omega)
$$

Nous appellerons $Z(j \omega)$ l'impédance du système d'adduction par analogie avec la loi d'OHM en électricité :

$\mathrm{E}\left(j_{\omega}\right)=\mathrm{I}\left(j_{\omega}\right) Z(j \omega)$ (Voir à ce sujet aussi le $\S$ III-3.)

L'orifice d'adduction établit la relation :

$$
h=\mathrm{K} \frac{q^{2}}{y^{2}}
$$

$\mathrm{K}$ : coefficient de perte de charge à pleine ouverture.

$y$ : ouverture relative de la vanne.

En linéarisant et posant :

$$
h=\mathcal{R}\left(\mathrm{H}_{0} e^{j_{\omega} t}\right) ; q=\mathcal{R}\left(\mathrm{Q}_{0} e^{j \omega t}\right)
$$

on obtient:

$$
\frac{\mathrm{H}_{0}}{\mathrm{H}}=\frac{2 \mathrm{Q}_{0}}{\mathrm{Q}}-\frac{2 y}{\mathrm{Y}}
$$

$\mathrm{H}:$ chute nette.

$\mathrm{Q}$ et $\mathrm{Y}$ : débit et ouverture de vanne en régime établi; $Q$ et $Y$ définissent le point de fonctionnement autour duquel on linéarise.

\footnotetext{
* $\mathcal{R}$ veut dire «partie réelle de... 》 (voir § III-4).
}

Compte tenu de $\mathrm{H}_{0}=\mathrm{Q}_{0} Z$, on obtient la fonction de transfert entre $y$ et $Q_{0}$.

$$
\frac{y}{\mathrm{Y}}=\frac{\mathrm{Q}_{0}}{\mathrm{Q}}\left(1-\frac{\mathrm{ZQ}}{2 \mathrm{H}}\right)
$$

Comme $\frac{Z Q}{H}$ intervient constamment, on pose:

$$
z=-\frac{\mathrm{ZQ}}{\mathrm{H}}
$$

En tenant compte de la fonction de transfert du bassin :

$$
\mathrm{S} j \omega \mathrm{N}_{0}=\mathrm{Q}_{0}
$$

on obtient :

$$
\frac{\mathrm{S}}{\mathrm{Q}} j(1)\left(1+\frac{z}{2}\right) \mathrm{N}_{0}=-\frac{y}{\mathrm{Y}}
$$

On peut écrire :

$$
z=\mathrm{X}(\omega)+j \mathrm{Y}(\omega)
$$

$\mathrm{X}$ est une fonction paire et $\mathrm{Y}$ une fonction impaire comme il ressort de l'article cité en [1]. Le déphasage dû à la partie linćaire est alors :

$$
-\pi / 2-\operatorname{arctg} \frac{\mathrm{Y}}{\mathrm{X}+2}
$$

Si l'installation est munie d'un régulateur par tout ou rien ordinaire, ce dernier produit un déphasage de :

$$
-\arccos \frac{4}{\pi} \frac{r_{0} m}{\omega|\mathrm{N}|}
$$

D'où la condition de cycle limite :

$$
\begin{aligned}
2 k \pi-\pi=-\pi / 2-\operatorname{arctg} & \frac{\mathrm{Y}}{\mathrm{X}+2} \\
& -\arccos \frac{4}{\pi} \frac{\mathrm{V}_{0} m}{\omega|\mathrm{N}|}
\end{aligned}
$$

Comme au $\S$ VI-11, on peut calculer l'amplitude du cycle limite éventuel en fonction de sa pulsation; on obtient :

$$
|\mathrm{N}|=\frac{4}{\pi} \frac{\mathrm{V}_{0}}{\omega} \frac{1}{\mathrm{~S} \omega} \frac{2 \mathrm{~K} \mathrm{Q}}{\mathrm{Y}} \frac{1}{\sqrt{(2+\mathrm{X})^{2}+\mathrm{Y}^{2}}}
$$

De ces deux équations on peut tirer l'équation des pulsations des cycles limites. Elle est :

$$
\left[(2+\mathrm{X})^{2}+\mathrm{Y}^{2}\right] \frac{\mathrm{S} m \mathrm{Y}}{2 \mathrm{Q}} \omega=\mathrm{Y}
$$

Nous voyons apparaître ici (comme dans tous les problèmes) la particularité suivante : on peut 
calculer la limite de stabilité quand le phénomène est oscillatoire, mais non quand il est apériodique. Or, physiquement, les régimes apériodiques ne sont à peu près jamais à craindre car il faut monter un élément « à l'envers » pour qu'ils puissent exister. Ce fait est d'ailleurs largement démontré pour les installations linéaires. Voir par exemple [6] où toutes les séparatrices $S_{0}$ et $\mathrm{S}_{\infty}$ sont des limites de «bon sens ». L'équation trouvée est vraiment « la » limite de stabilité.

Nous allons rapidement étudier cette équation d'une facon générale. Admettons d'abord que l'adduction soit un système à constante localisée, c'est-à-dire que les phénomènes d'ondes soient négligeables (cf. §III-3). L'équation en $\omega$ est alors algébrique et les cycles limites sont donnés par les racines réelles et positives de cette équation. Comme $\mathrm{X}$ et $\mathrm{Y}$ sont des expressions respectivement paire et impaire en $\omega$, l'équation est en $(j \omega)^{2}$ ou en $\omega^{2}$ et ses coefficients sont tous réels. Si on pose $\omega^{2}=u$, on obtient une nouvelle équation $f(u)=0$ que nous appellerons équation caractéristique. Les cycles limites sont donnés par les racines réelles et positives de cette équation caractéristique. Si on étudie une installation dans laquelle un certain nombre de grandeurs (ou toutes les grandeurs) ne sont pas déterminées numériquement, les coefficients des termes $u^{m}$ de l'équation en $u$ seront fonction de ces grandeurs. Nous appellerons ces grandeurs les paramètres du système. Le nombre de racines réelles ou positives de $f(u)=0$ dépend alors de la valeur de ces paramètres. On peut ici faire une discussion analogue à celle qui a été faite pour les systèmes linéaires*. On peut considérer un espace dont les axes (ou plutôt les surfaces coordonnées) sont gradués d'après les valeurs des paramètres. S'il y a $k$ paramètres, l'espace aura $k$ dimensions. Dans cet espace on peut tracer des hypersurfaces limitant des domaines dans lesquels le nombre de racines réelles et positives de l'équation caractéristique reste constant. Ces hypersurfaces seront appelées (en analogie avec les systèmes linéaires): séparatrices **. Le nombre de racines réelles et positives peut varier si une racine passe par la valeur zéro ou la valeur infinie, ou si deux racines réelles se confondent pour donner ensuite deux racines imaginaires. Il y a done trois hypersurfaces séparatrices: $\mathrm{S}_{0}$, lieu des points où $f(u)=0$ a une racine nulle; $S_{\infty}$, lieu des points oli $f(u)=0$ a une racine infinie, et $S_{2}$, lieu des points où $f(u)=0$ a une racine double. Pour plus de détails sur ce genre de raisonnement, on peut voir [7] où est traité le cas des systèmes linéaires.

\footnotetext{
* Voir [7].

* Voir [5] et [7].
}

Etudions ici plus en détail simplement le cas d'un petit nombre de paramètres, dont deux interviennent linéairement dans $f(u)=0^{\star}$. On peut évidemment faire des études analogues pour des cas bien moins élémentaires, mais les notions mathématiques mises en jeu deviennent rapidement de plus en plus compliquées. Les quelques détails que nous indiquons ici seront présentés aussi simplement que possible, bien qu'ils fassent déjà appel à des notions mathématiques bien plus élevées que l'ensemble de notre étude.

\section{Soit donc:}

$$
f(u)=a_{n} u^{n}+a_{n-1} u^{n-1}+\ldots+a_{1} u+a_{0}=0
$$

la fonction caractéristique à étudier et les $a_{n}$ fonctions de plusieurs paramètres dont 2 : $\lambda$ et $u$. interviennent uniquement linéairement. Nous écrirons :

$$
f(u)=\varphi(u ; \lambda ; \mu)=0
$$

Nous allons étudier ce qui se passe dans le plan $\lambda ; \mu$ (qui est un espace des paramètres particulier) quand on maintient les autres parametres fixes. Les valeurs de $\lambda$ et $u$ pour lesquelles $f(u)=\varphi(u ; \lambda ; \mu)=0$ a une racine égale à $u_{0}$ sont sur la droite $\varphi\left(u_{0} ; \lambda ; \mu\right)=0$. Nous appellerons cette droite $\Delta\left(u_{0}\right)$. Par chaque point du plan passent $n$ droites relatives aux $n$ racines $u$ de l'équation $f(u)=0$. Le nombre de droites réelles qui passent en chaque point donne le nombre de racines réelles de l'équation $f(u)=0$. Appelons l'enveloppe des droites $\Delta\left(u_{0}\right)$ : la courbe $C$. Cette courbe $C$ est le lieu des points pour lesquels l'équation caractéristique a une racine double, puisque sur cette courbe $\varphi\left(u_{0} ; \lambda ; \mu\right)=0$ et $\varphi^{\prime}{ }_{u_{0}}\left(u_{0} ; \lambda ; \mu\right)=0$. La courbe $\mathrm{C}$ est donc la séparatrice $S_{2}$. Elle est unicursale puisqu'on peut la paramétrer en $u_{0}$ en tirant $\lambda$ et $u$. des deux équations linéaires $\varphi=0$ et $\varphi^{\prime}{ }_{u_{0}}=0$. Son degré est $2(n-1)$ : on peut en effet remplacer $\varrho$ par la combinaison $n \varphi-u_{0} \varphi^{\prime} u_{0}=0$, d'où deux équations du $(n-1)^{\circ}$ degré en $u_{0}$ (on ne peut pas abaisser plus le degré ). Le nombre de points doubles d'une courbe unicursale de degré $d$ étant $\frac{(d-1)(d-2)}{2}$, la courbe $\mathrm{C}$ a $(2 n-3)(n-2)$ points doubles. Le paramètre $u_{0}$ de la courbe $\mathrm{C}$ est précisément la racine double de l'équation $\varphi=0$. Aux points doubles de la courbe $\mathrm{C}$ correspondent des valeurs $\lambda$ et $\mu$ pour lesquelles l'équation caractéristique a deux racines doubles. Aux points de rebroussement correspondent des racines triples: Les racines triples sont données par les trois équations :

* On peut ramener un très grand nombre de cas pratiques à cette forme. 


$$
\begin{gathered}
\varphi\left(u_{0} ; \lambda ; \mu\right)=0 ; \quad \varphi^{\prime}{ }_{u_{0} 0^{2}}\left(u u_{0} ; \lambda ; \mu\right)=0 \\
\text { et } \varphi^{\prime \prime}{ }_{u_{0}^{2}}\left(u_{0} ; \lambda ; \mu\right)=0
\end{gathered}
$$

Comme pour les points doubles, on peut remplacer ces trois équations par trois combinaisons linéaires de degré $(n-2)$ en $u$. On peut éliminer $\lambda$ et $\mu$ en annulant le déterminant complet du système. On en tire une équation de degré $3(n-2)$ en $u$. Comme à chaque valeur $u$ correspond une valeur $\lambda$ et une valeur $\mu$, la courbe $C$ a $3(n-2)$ points de rebroussement. Il reste donc $2(n-3)(n-2)$ points doubles ordinaires. On vérifie aisément que la classe de la courbe $C$ est bien $n$ *. On appelle classe d'une courbe algébrique le nombre de tangentes (réelles ou imaginaires) qu'on peut y mener par un point. On peut évidemment mener $n$ tangentes à la courbe $\mathrm{C}$; ces $n$ tangentes sont les $n$ droites $\Delta\left(u_{0}\right)$ passant par le point considéré. Le nombre de racines réelles de l'équation caractéristique est égal au nombre de tangentes réelles qu'on peut tracer par le point correspondant de la séparatrice $S_{2}$. Les séparatrices $S_{0}$ et $S_{\infty}$ sont dans notre cas les droites $a_{0}=0$ et $a_{n}=0$. Ce sont des droites $\Delta\left(u_{0}\right)$, donc des tangentes à $S_{2}$.

On remarque qu'il suffit de tracer la courbe $C$ pour les valeur's du paramètre $u_{0}$ telles que $0 \leqslant u_{0} \leqslant \infty$. Cet arc et les droites $a_{0}=0$ et $a_{n}=0$ résolvent entièrement notre problème.

Résumons en quelques mots :

Si l'équation caractéristique est de degré $n$ et contient linéairement deux paramètres $\lambda$ et $\mu$, la séparatrice $\mathrm{S}_{2}$ est, dans le plan $\lambda ; u$, une courbe unicursale, c'est-à-dire paramétrable. Cette courbe est du degré $2 n-2$; elle a $3(n-2)$ points de rebroussement et $2(n-3)(n-2)$ points doubles ordinaires. Les points doubles fournissent les valeurs de $\lambda$ et $\mu$ pour lesquelles l'équation caractéristique a deux racines doubles; les points de rebroussement fournissent les racines triples. Le nombre de racines réelles de l'équation caractéristique est égal au nombre de tangentes réelles qu'on peut mener du point correspondant à la séparatrice $S_{2}$. Les séparatrices $S_{0}$ et $S_{\alpha}$ sont des droites tangentes à $S_{2}{ }^{* *}$.

Etudions maintenannt rapidement ce qui se passe si on remplace le régulateur par tout ou rien par le régulateur infiniment lent dont il a été question plus haut. Il faut joindre à :

$$
\mathrm{S} j \omega \cdot\left(1+\frac{z}{2}\right) \frac{\mathrm{N}_{0}}{\mathrm{Q}}=\frac{y}{\mathrm{Y}}
$$

* La classe d'une courbe de degré $d$ et ayant $p$ points doubles et $r$ points de rebroussement est $d(d-1)-2 p-3 r$.

** L'equation $f(u)$ peut évidemment aussi servir dans les cas entièrement numériques. On trace alors effectivement $y=f(u)$ pour $u>0$ et on vérifie qu'il n'existe pas de racine réelle. la fonction de transfert:

avec :

$$
\left(j \omega+\mathrm{K}_{0} m\right) y=-\mathrm{K}_{0} \mathrm{~N}_{0}
$$

D’où l'équation :

$$
K_{0} \rightarrow 0
$$

$$
\mathrm{S} j \omega\left(j \omega+\mathbf{K}_{0} m\right)\left(1+\frac{z}{2}\right)+\frac{K_{0}}{\mathrm{Y}}=0
$$

Pour abréger les calculs, plaçons-nous dans le cas où les pertes de charge sont négligeables ou équivalentes à une perte de charge s'ajoutant à celle de l'orifice et où $z(j \omega)$ est un polynôme. Le domaine de stabilité est alors limité par $\Delta_{n-1}=0 ; \Delta_{n-1}$ est le déterminant d'HuRwiTz dont l'ordre est d'une unité plus petit que l'ordre de l'équation caractéristique. (Voir [7].) Les coefficients des puissances $0 ; 1 ; 4 ; 6 \ldots 2 n$ en $j \omega$ sont proportionnelles à $K_{0}$, les autres contiennent des termes indépendants de $K_{0}$ (en effet, $z(j \omega)$ ne contient dans ce cas que des puissances impaires de $j \omega)$. Si $\mathrm{K}_{0} \rightarrow 0$, on peut écrire l'équation caractéristique :

$$
\begin{gathered}
\mathrm{K}_{0} a_{0}+\mathrm{K}_{0} a_{1} j \omega+a_{2}(j \omega)^{2}+a_{3}(j \omega)^{3} \\
+\mathrm{K}_{0} a_{4}(j \omega)^{+}+a_{5}(j \omega)^{5}+\ldots
\end{gathered}
$$

Le déterminant $\Delta_{n-1}$ sera :

\begin{tabular}{|ccccccc}
$\mathrm{K}_{0} a_{1}$ & $\mathrm{~K}_{0} a_{0}$ & 0 & 0 & 0 & 0 & $\cdots$ \\
$a_{3}$ & $a_{2}$ & $\mathrm{~K}_{0} a_{1}$ & $\mathrm{~K}_{0} a_{0}$ & 0 & 0 & \\
$a_{5}$ & $\mathrm{~K}_{0} a_{4}$ & $a_{3}$ & $a_{2}$ & $\mathrm{~K}_{0} a_{1}$ & $\mathrm{~K}_{0} a_{0}$ & $\cdots$ \\
$a_{7}$ & $\mathrm{~K}_{0} a_{6}$ & $a_{5}$ & $\mathrm{~K}_{0} a_{4}$ & $a_{3}$ & $a_{2}$ & \\
$a_{9}$ & $\mathrm{~K}_{0} a_{7}$ & $a_{7}$ & $\mathrm{~K}_{0} a_{6}$ & $a_{5}$ & $\mathrm{~K}_{0} a_{1}$ &
\end{tabular} \mid

On ne peut mettre $K_{0}$ en facteur que dans la première ligne. Si $\mathrm{K}_{0} \rightarrow 0$, on peat supprimer tous les autres termes en $\boldsymbol{K}_{0}$ et le domaine de stabilité est limité par le déterminant :

$$
\left|\begin{array}{lllllllll}
a_{1} & a_{0} & 0 & 0 & 0 & 0 & 0 & 0 & \cdots \\
a_{3} & a_{2} & 0 & 0 & 0 & 0 & 0 & 0 & \\
a_{5} & 0 & a_{3} & a_{2} & 0 & 0 & 0 & 0 & \\
a_{7} & 0 & a_{5} & 0 & a_{3} & a_{2} & 0 & 0 & \\
a_{9} & 0 & a_{7} & 0 & a_{5} & 0 & a_{3} & a_{2} &
\end{array}\right|
$$

On peut le développer par rapport à la première ligne d'où $\Delta_{n-1}=\left(a_{1} a_{2}-a_{0} a_{3}\right) \Delta^{\prime}$ où $\Delta^{\prime}$ est le déterminant :

$$
\left|\begin{array}{llllllll}
a_{3} & a_{22} & 0 & 0 & 0 & 0 & 0 & \cdots \\
a_{5} & 0 & a_{3} & a_{2} & 0 & 0 & 0 & \\
a_{7} & 0 & a_{5} & 0 & a_{3} & a_{2} & 0 & \\
a_{9} & 0 & a_{7} & 0 & a_{5} & 0 & a_{3} & \\
a_{11} & 0 & a_{9} & 0 & a_{5} & 0 & a_{5} &
\end{array}\right|
$$

Ce nouveau déterminant peut être développé par rapport à tous les coefficients $a_{2}$. Ceci revient à rayer la moitié des lignes. On sait que dans la moitié inférieure du déterminant $\Delta_{n-1}$ il existe un triangle de zéros. Après avoir rayé les colonnes des $a_{2}$, il resté un déterminant diagonal dont la valeur est $a_{n}{ }^{m}$. Comme $a_{2}$ et $a_{n}$ sont nécessairement positifs, la limite de stabilité est $a_{1} a_{2}=a_{0} a_{3}$. 
Si on développe $z(j \omega)$ en :

$$
z(j \omega)=\mathrm{A}+\mathrm{B} j \omega+\mathrm{C}(j \omega)^{3}+\mathrm{D}(j \omega)^{5}+\ldots
$$

La limite de stabilité est donnée par :

$$
\mathrm{S} m \mathrm{Y}(2+\mathrm{A})^{2}=\mathrm{B} \mathrm{Q}
$$

Seuls A et B interviennent, alors que dans le cas du régulateur par tout ou rien tous les coefficients interviennent. C'est seulement dans le cas où tous, sauf $\mathrm{A}$ et $\mathrm{B}$, sont nuls (ou n'interviennent pas), que les deux régulateurs peuvent donner la même condition de stabilité. Il se trouve qu'elle est alors effectivement la même : ceci a seulement lieu pour une installation dont l'adduction se fait par une conduite unique à coups de bélier en masse.

Etudions ici rapidement ce cas important d'une façon générale (le cas sans pertes de charge a déjà été traité au §VI-11).

On a :

$$
\mathrm{A}=2 \Delta \mathrm{H} \frac{\mathrm{Q}^{2}}{\mathrm{Q}_{m}{ }^{i 2}} \quad ; \quad \mathrm{B}=\Theta=\frac{\mathrm{L}}{g_{\sigma}} \frac{\mathrm{Q}}{\mathrm{H}_{\mathrm{s}}}
$$

$\Delta H$ : perte de charge dans la conduite au débit maximum $\mathrm{Q}_{m}$.

Les autres notations sont celles du § VI-11.

En raison des pertes de charge de la conduite, $Q$ et $\mathrm{Y}$ ne sont pas proportionnels mais reliés par :

$$
\mathrm{Y}=\frac{\mathrm{Q}}{\mathrm{Q}_{m}} \sqrt{\frac{\mathrm{H}_{s}-\Delta \mathrm{H}}{\mathrm{H}_{s}-\Delta \mathrm{H}\left(\mathrm{Q}^{2} / \mathrm{Q}_{m}^{2}\right)}}
$$

D'où la condition de stabilité :

$$
\left(1+\frac{\Delta \mathrm{H}}{\mathrm{H}_{s}} \frac{\mathrm{Q}^{2}}{\mathrm{Q}_{m}{ }^{2}}\right) \frac{2 m \mathrm{~S}}{\mathrm{Q}_{m}} \sqrt{\frac{1-\frac{\Delta \mathrm{H}}{\mathrm{H}_{s}}}{1-\frac{\Delta \mathrm{H}}{\mathrm{H}_{s}} \frac{\mathrm{Q}^{2}}{\mathrm{Q}_{m}{ }^{2}}}}>\frac{\mathrm{L}}{g \sigma} \frac{\mathrm{Q}}{\mathrm{H}_{s}}
$$

Pour trouver le débit le plus défavorable, il faut chercher le minimum de :

$$
y=\frac{(1+x)^{2}}{x^{2}(1-x)} \quad \text { ò̀ } \quad x=\frac{\Delta \mathrm{H}}{\overline{\mathrm{H}}_{s}} \frac{\mathrm{Q}}{\mathrm{Q}_{m}}
$$

on trouve : $x=1 / 3$.

Donc l'installation est la moins stable pour :

$$
\mathrm{Q}=\mathrm{Q}_{m} \sqrt{\frac{\mathrm{H}_{s}}{3 \Delta \mathrm{H}}} \quad \text { si } \quad \Delta \mathrm{H}>\frac{\mathrm{H}_{s}}{3}
$$

et :

$$
Q=Q_{m} \quad \text { si } \quad \Delta H<\frac{H_{s}}{3}
$$

Il faut donc que la grandeur :

$$
b=\frac{2 m \mathrm{~S}}{\mathrm{Q} \Theta_{m}}\left(\text { où } \Theta_{m}=\frac{\mathrm{L}}{g \sigma} \frac{\mathrm{Q}_{m}}{\mathrm{H}_{s}}\right)
$$

respecte la condition :

$$
\begin{aligned}
\quad b>\frac{1}{2} & \frac{\mathrm{H}_{s}}{\sqrt{2 \Delta \mathrm{H}\left(\mathrm{H}_{s}-\Delta \mathrm{H}\right)}} \text { pour } \Delta \mathrm{H}>\frac{\mathrm{H}_{s}}{3} \\
\text { ou }: \quad b & >\frac{1}{1+\frac{\Delta H}{\mathrm{H}_{s}}} \text { pour } \Delta \mathrm{H}<\frac{\mathrm{H}_{s}}{3}
\end{aligned}
$$

La fonction $b=b \frac{\Delta \mathrm{H}}{\mathrm{H}_{s}}$ est représentée sur la figure 19. La perte de charge est fonction, entre

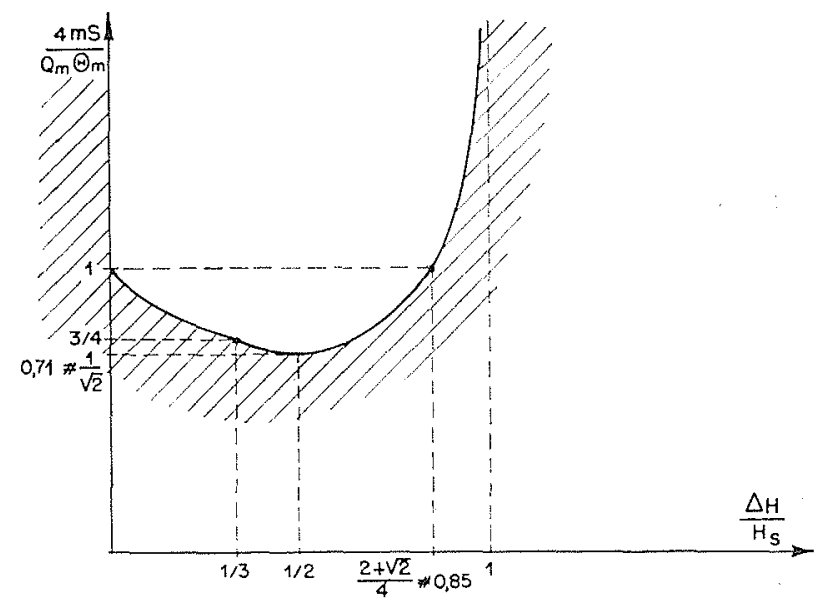

Frg. 19. - Le domaine de stabilité est en blanc.

autre, du diamètre de la conduite; mais comme le diamètre intervient dans $\sigma$, la figure 19 ne traduit pas exactement les variations de stabilité en fonction du diamètre. Si les pertes de charge sont inversement proportionnelles à la puissance $5^{\circ}$ du diamètre (comme on l'admet souvent), l'optimum est atteint si la perte de charge est $(11 / 16) \mathrm{H}_{s}$; mais comme la courbe est fortement croissante entre $11 / 16$ et 1 , il vaut mieux choisir une valeur un peu en dessous de $11 / 16$, à cause de l'imprécision avec laquelle on connaît $\Delta \mathrm{H}$.

\section{VI-21. - Régulateur par tout ou rien et adduction par conduite unique à coups de bélier en onde (Fig, 20)}

Nous étudierons le cas où les pertes de charge sont négligeables. Le coup de bélier dans la con- 


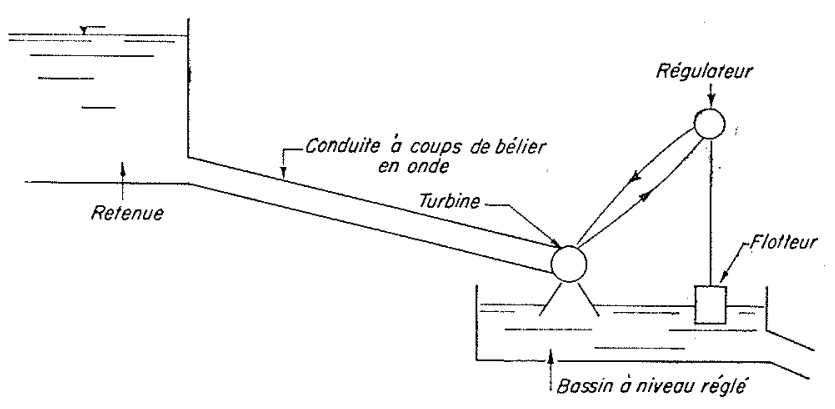

FIG. 20

duite est régi par les équations (voir par exemple [2]):

$$
\begin{gathered}
\frac{1}{\sigma} \frac{\partial q}{\partial t}=-g \frac{\partial h}{\partial x} \\
\frac{g \sigma}{\alpha^{2}} \frac{\partial h}{\partial t}+\frac{\partial q}{\partial x}=0
\end{gathered}
$$

$x$ : abscisse le long de la conduite.

$q$ : débit dans le sens des $x$ croissants au point $x$ et à l'instant $t$.

$h$ : hauteur au point $x$ et à l'instant $t$.

$g: 9,81 \mathrm{~m} / \mathrm{sec}^{2}$ : accélération de la pesanteur.

$a$ : célérité des ondes de coups de bélier.

$\sigma$ : section de la conduite.

En amont, on a :

$h=0$ (définition du plan de référence des charges).

Pour étudier les régimes sinusoïdaux, il suffit de poser :

$$
\begin{aligned}
& q=\mathcal{R}\left[\mathrm{Q}_{0}(x) e^{j \omega t}\right]+\mathrm{Q} \\
& h=\mathcal{R}\left[\mathrm{H}_{0}(x) e^{j \omega t}\right]+\mathrm{H}
\end{aligned}
$$

$\mathrm{H} ; \mathrm{Q}$ : hauteur et débit en régime permanent au point de fonctionnement étudié.

On en déduit :

$$
\begin{gathered}
(1 / \sigma) j \omega \mathrm{Q}_{0}(x)=-g \mathrm{H}_{0, x}^{\prime}(x) \\
\left(g \sigma / a^{2}\right) j \omega \mathrm{H}_{0}(x)+\mathrm{Q}_{0, x}^{\prime}(x)=0
\end{gathered}
$$

d'où :

$$
\mathrm{H}_{0}(x)=\mathrm{A} \cos \frac{\omega}{a} x+\mathrm{B} \sin \frac{\omega}{a} x
$$

où $\mathrm{A}$ et $\mathrm{B}$ sont des constantes d'intégration fonctions de $\omega$ mais indépendantes de $x$.
La condition amont se traduit par $\mathrm{A}=0$, d'où:

$$
\begin{gathered}
\mathrm{H}_{0}(x)=\mathrm{B} \sin \frac{\omega}{a} x \\
\text { et : } \quad \mathrm{Q}_{0}(x)=\frac{g \sigma}{a} j \mathrm{~B} \cos \frac{\omega}{a} x
\end{gathered}
$$

L'impédance est :

$$
\begin{gathered}
\mathrm{Z}=-j \frac{\Theta}{0} \frac{\mathrm{H}}{\mathrm{Q}} \operatorname{tg} \omega \theta \\
\Theta=\frac{\mathrm{L}}{g \sigma} \frac{\mathrm{Q}}{\mathrm{H}} \quad ; \quad \theta=\frac{\mathrm{L}}{a}
\end{gathered}
$$

d'où l'équation en $\omega$ :

$$
\frac{\mathrm{S} m \mathrm{Y}}{2 \mathrm{Q} \Theta}\left(4+\frac{\Theta^{2}}{0^{2}} \operatorname{tg}^{2} \omega \theta\right)=\frac{1}{\theta \omega} \operatorname{tg} \omega \theta
$$

On peut poser :

$$
\frac{2 \mathrm{Q} \Theta}{\mathrm{S} m \mathrm{Y}}=b \quad \text { et } \quad \frac{\Theta^{2}}{\theta^{2}}=c
$$

Dans le cas présent, l'équation en $\omega$ n'est pas algébrique, néanmoins on peut la discuter facilement. Posons :

$$
\omega \theta=u
$$

D'où :

$$
f(u)=4 u+c u \operatorname{tg}^{2} u-b \operatorname{tg} u=0
$$

La séparatrice $\mathrm{S}_{2}$ est donnée par :

$$
f^{\prime}(u)=4+c\left(\operatorname{tg}^{2} u+\frac{2 u \operatorname{tg} u}{\cos ^{2} u}\right)-\frac{b}{\cos ^{2} u}=0
$$

On en tire :

$$
\begin{aligned}
& b=\frac{8 u^{2}}{\sin ^{2} u+u \operatorname{tg} u} \\
& c=2 \frac{2 u-\sin 2 u}{\operatorname{tg} u\left(\sin ^{2} u+u \operatorname{tg} u\right)}
\end{aligned}
$$

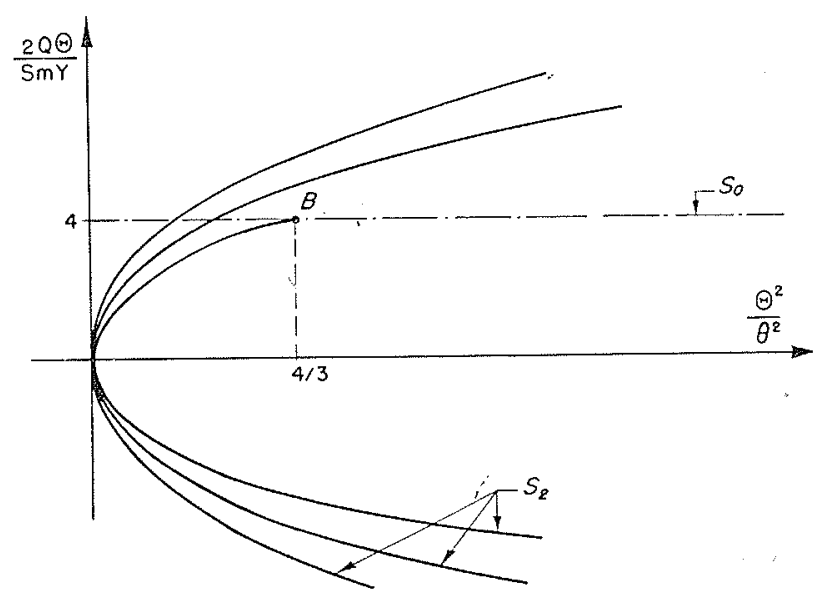

FIG. 21 
On peut facilement construire $b(u)$ et $c(u)$. On en déduit l'allure de la courbe $S_{2}$ dans le plan $b ; c$ (fig. 21). Les points correspondants à $u=u_{0}$ et $u=-u_{0}$ sont confondus. $u=0$ est donc un point d'arrêt. $S_{0}$ est la droite $b=4$. Du domaine compris entre l'axe $o c$, la droite $\mathrm{S}_{0}$ et la branche de $\mathrm{S}_{2}$ débutant en $c=4 / 3 ; b=4$, on ne peut mener aucune tangente à la partie de la courbe $S_{2}$ tracée. Dans ce domaine il ne saurait y avoir de cycle limite, il y a stabilité pure et simple. On remarque que $c=4 p^{2}$ où o est le paramètre bien connu d'Allievi $\left(?=\frac{a \mathrm{~V}}{g \mathrm{H}}\right)$.

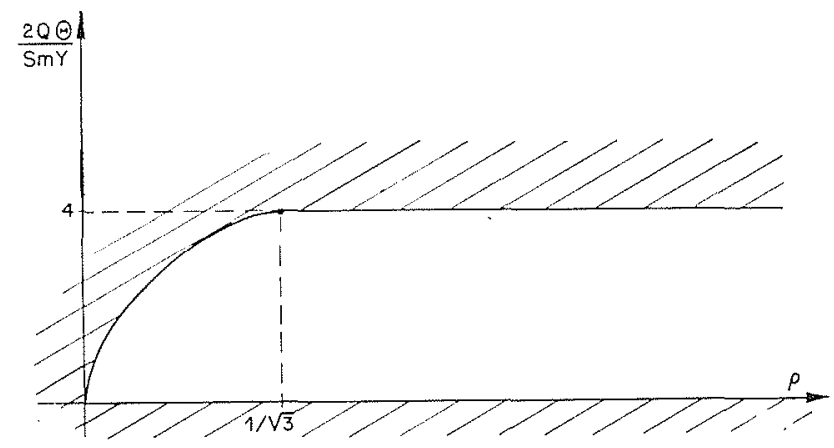

Frg. 22. - Le domaine de stabilité est en blanc.

L'approximation du coup de bélier en masse est donc rigoureusement valable ici jusqu'à $\rho=\frac{1}{\sqrt{3}} \# 0,56$. Si $\imath<\frac{1}{\sqrt{3}}$, le domaine de stabilité est moins grand *.

\section{VI-22. - Régulateur par tout ou rien et adduction}

comprenant une cheminée d'équilibre (Fig. 23)
Les autres symboles ont la même signification que plus haut. Nous négligerons les pertes de charge de l'adduction.

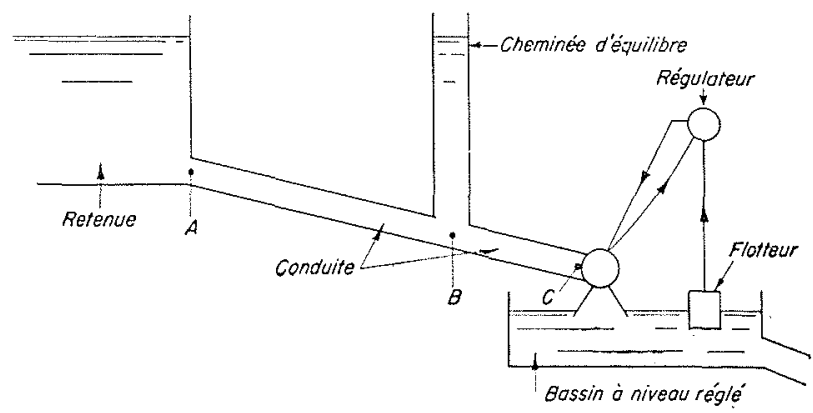

FIG. 23

On a les équations :

$$
\begin{aligned}
& \mathrm{H}_{s}-\frac{\mathrm{L}}{g \sigma} \frac{d q_{1}}{d t}=\mathrm{H}_{c} \\
& \mathrm{~S} \frac{d \mathrm{H}_{c}}{d t}=q_{: 3} \\
& q_{2}=q_{1}-q_{: 3} \\
& \mathrm{H}_{c}-\frac{\mathrm{L}_{2}}{g \sigma_{2}} \frac{d q_{2}}{d t}=\mathrm{H}
\end{aligned}
$$

On pose :

$\Theta_{1}=\frac{\mathrm{L}_{1}}{g \sigma_{1}} \frac{\mathrm{Q}}{\mathrm{H}_{s}} ; \quad \Theta_{2}=\frac{\mathrm{L}_{2}}{g \sigma_{2}} \frac{\mathrm{Q}}{\mathrm{H}_{s}} ; \mathrm{T}=\frac{\mathrm{SQ}}{\mathrm{H}_{s}}$

Après linéarisation, on en tire l'impédance :

$$
\mathrm{Z}(j \omega)=-\frac{\mathrm{H}}{\mathrm{Q}} \frac{1+\frac{\Theta_{2}}{\Theta_{1}}-\mathrm{T} \Theta_{2} \omega^{2}}{\mathrm{~T} j \omega+\frac{1}{\Theta_{1} j \omega}}
$$

d'où l'équation en $\omega$ :

$\mathrm{L}_{1}$ : longueur du tronçon A B.

$\sigma_{1}$ : section du tronçon $\mathrm{A} B$.

$\mathrm{L}_{2}$ : longueur du tronçon B C.

$\sigma_{2}:$ section.

$q_{1}$ : débit dans A B.

$q_{2}:$ débit dans $\mathrm{B}$ C.

$q_{3}$ : débit montant dans la cheminée.

$$
\begin{aligned}
\left(1+\frac{\Theta_{2}}{\Theta_{1}}-\mathrm{T} \Theta_{2} \omega^{2}\right)( & \left.-\mathrm{T} \omega+\frac{1}{\Theta_{1} \omega}\right)=\left[4\left(\mathrm{~T} \omega-\frac{1}{\Theta_{1} \omega}\right)^{2}\right. \\
& \left.+\left(1+\frac{\Theta_{2}}{\Theta_{1}}-\mathrm{T} \Theta_{2} \omega^{2}\right)^{2}\right] \frac{\mathrm{S} m \mathrm{Y}}{2 \mathrm{Q}} \omega
\end{aligned}
$$

On pose :

$$
\lambda=\frac{\mathrm{T}}{4 \Theta_{1}} ; u=\omega^{2} \Theta_{1} \mathrm{~T} ; \mu=\frac{2 \mathrm{Q} \mathrm{T}}{m \mathrm{ST}} ; c=\frac{\Theta_{2}}{\Theta_{1}}
$$

d'où :

$$
\begin{aligned}
f(u)= & u^{3} c^{2}-u^{2}[2 c(c+1)-\lambda+u c] \\
+u\left[(c+1)^{2}-2 \lambda\right. & +\mu(1+2 c)] \\
& +\lambda-\mu(1+c)=0
\end{aligned}
$$


Pour trouver la séparatrice $S_{2}$ qui est un lieu de points doubles, on peut faire un changement de variable; on pose $u-1=v$ et on obtient :

$$
\begin{aligned}
v^{3} c^{2}+v^{2}\left(c^{2}-2 c+\lambda-c(\mu)\right. & \\
& +v(1-2 c+\mu)+1=0
\end{aligned}
$$

On peut également faire le changement d'axe :. $+1=v$.

\section{D'où :}

$$
\begin{aligned}
\varphi(v)=v^{3} c^{2}+v^{2}\left(c^{2}-c+\lambda-c \nu\right) \\
\quad-v(2 c-\nu)+1=0
\end{aligned}
$$

La séparatrice $\mathrm{S}_{2}$ est alors donnée par :

$$
\begin{aligned}
& \nu=\frac{c^{2} v^{3}+2 c v-2}{v} \\
& \lambda=\frac{c^{3} v^{4}-2 c^{2} v^{3}+v^{2} c(c+1)-2 c v+1}{v^{2}}
\end{aligned}
$$

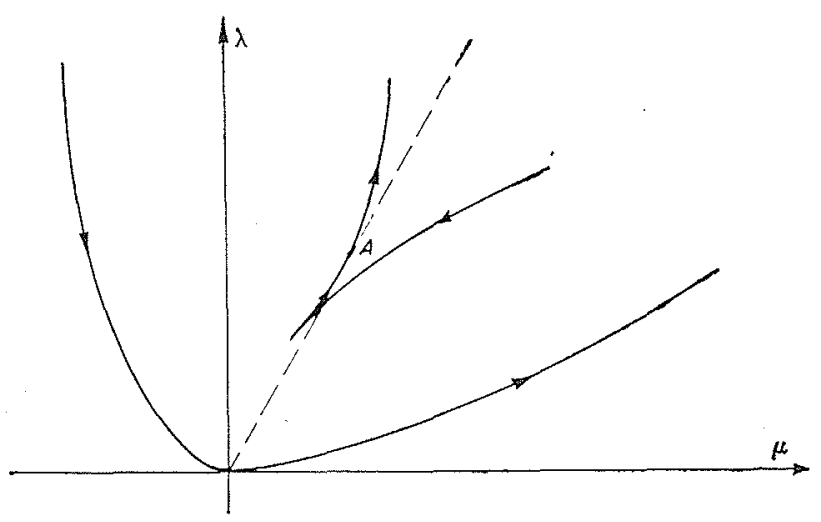

FIG. 24

L'allure de cette courbe dans le plan $\lambda ;$ p. est présentée sur la figure 24.

Les flèches indiquent le sens du parcours quand $u$ croît. Le point A correspond ’̀ $u=0$. La tangente en $A$ est la séparatrice $S_{0}$. On voit sur l'équation en $u$ que $S_{0}$ passe par l'origine. Pour obtenir les domaines sans cycles limites,

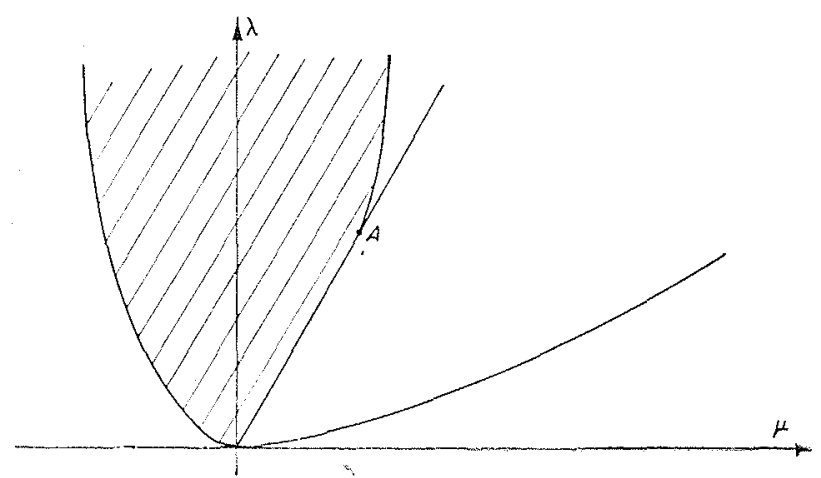

Frg. 25 on peut supprimer la partie de $S_{2}$ qui correspond à $u<0$; les tangentes à cette partie de la courbe donnent des racines $u$ négatives. D'où la limitation effective des domaines d'après le nombre de cycles limites possibles (fig. 25). A l'intérieur du domaine hachuré se trouvent les points d'où on ne peut mener ancune tangente a la partie restante de la courbe $S_{2}$. En réalité, si $\mu<0$, il y a instabilité apériodique. Le domaine de stabilité est donné par la figure 26.

En réalité, les paramètres :

$$
x=\frac{4 \mu(1+c)}{\lambda}=\frac{2 Q\left(\Theta_{1}+\Theta_{2}\right)}{m S Y} \text { et } y=\lambda
$$

sont plus intéressants que $\mu$ et $\lambda$. La figure 27 montre le domaine de stabilité dans le plan de ces paramètres.

On voit qu'il existe un domaine de stabilité: de plus, la cheminée est sans influence sur la limite de stabilité tant que sa section reste in-

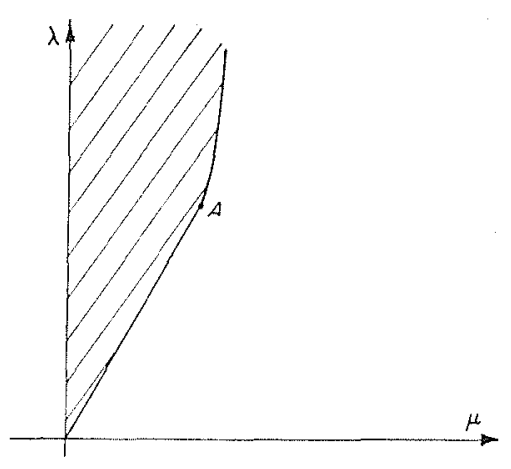

FIG. 26

férieure à une valeur critique. La cheminée a, par contre, toujours une influence sur la fréquence du cycle si la condition de stabilité n'est pas satisfaite.

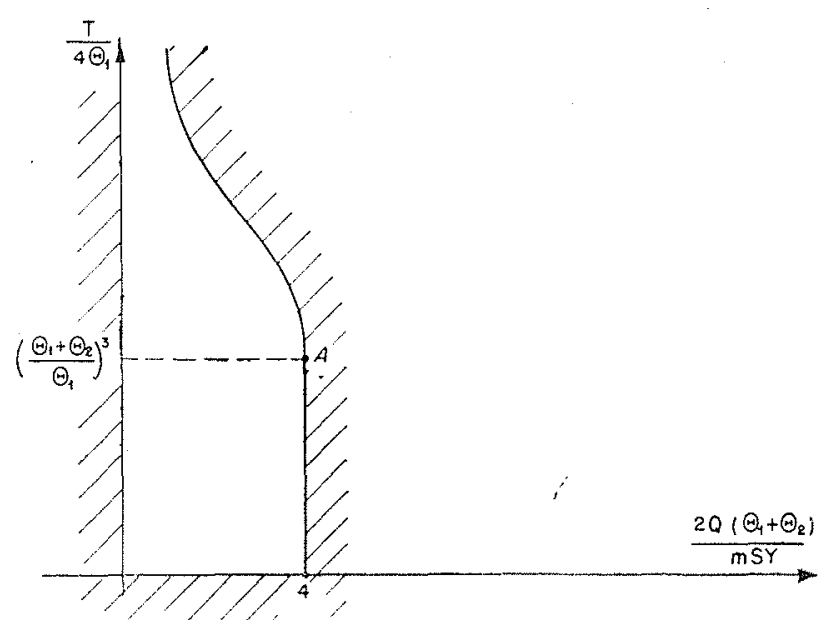

Frc. 27. - Le domaine de stabilite est en blanc. 
On voit ici la grande différence entre les régulateurs à vitesse proportionnelle à l'écart et les régulateurs par tout on rien : le système correspondant à la figure 23 et muni d'un régulateur à vitesse proportionnelle à l'écart n'est jamais stable s'il est sans perte de charge.

\section{VI-23. - Régulateur à vitesse proportionnelle à l'écart et liaison à jeu}

Dans ce cas, une partie de l'installation est linéaire (ou sera considérée comme telle) et une partie «algébrique » (voir \& V-234). On peut employer la méthode inventée par M. Kochenbur(iER et DUTruh (voir [3] et [4]).

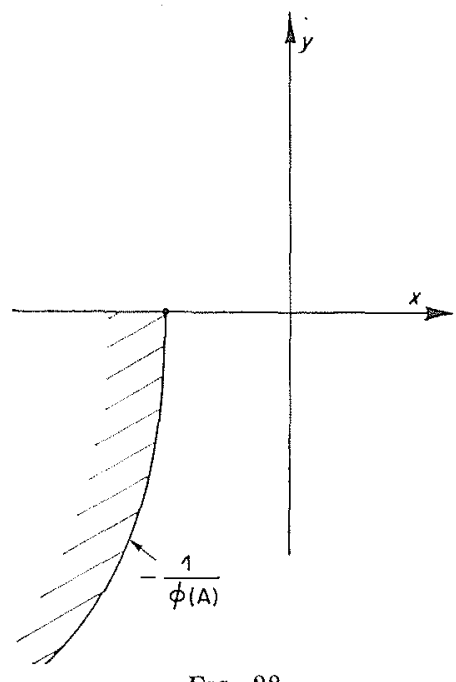

FIG. 28

La fonction de transfert globale est de la forme :

$$
\mathbf{F}(j \omega) \Phi(\mathrm{A})
$$

II y a cycle limite si :

$$
F(j \omega) \cdot \Phi(A)=-1
$$

oul :

$$
F(j \omega)=-\frac{1}{\Phi(\mathrm{A})}
$$

Si l'élément non linéaire est donné, la courbe -1/ 1 (A) est fixe dans le plan de Nyouis' (fig. 28), quand on fait varier les paramètres de la partie linéaire de l'installation.

Il y a stabilité si la courbe de transfert $F(j \omega)$ ne coupe pas la courbe - 1/ $\Phi(A)$ : done cette courbe ne pénètre pas dans le domaine hachuré.
La limite de stabilité est donnée par la condition de tangence entre $\mathrm{F}(j \omega)$ et $-1 / \Phi(\mathrm{A})$. Pratiquement, cela revient à "épaissir » le point - 1 et à en faire une zone (fig. 29). En général, les élé-

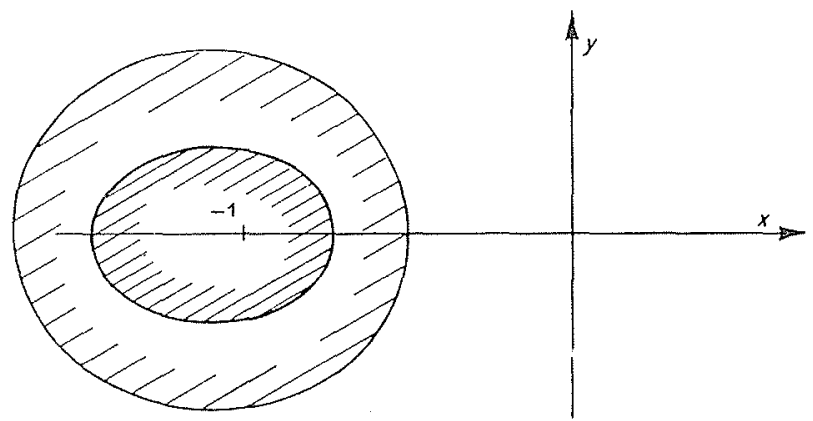

FIG. 29

ments algébriques considérés ainsi sont des « imperfections » comme les jeux, les frottements, etc. Les zones ainsi tracées jouent le rôle du point -1 pour les systèmes entièrement linéaires. La graduation de la courbe limite n'intervient pas.

Etudions ici simplement un cas schématique. Nous admettrons que la courbe de transfert d'un jeu soit un demi-cercle. Sur la figure 30 , on a représenté le demi-cercle et la courbe plus exacte (voir \& V-232). On voit que l'approximation n'est pas mauvaise, surtout compte tenu de l'imprécision avec laquelle on connaît en général les «imperfections ».

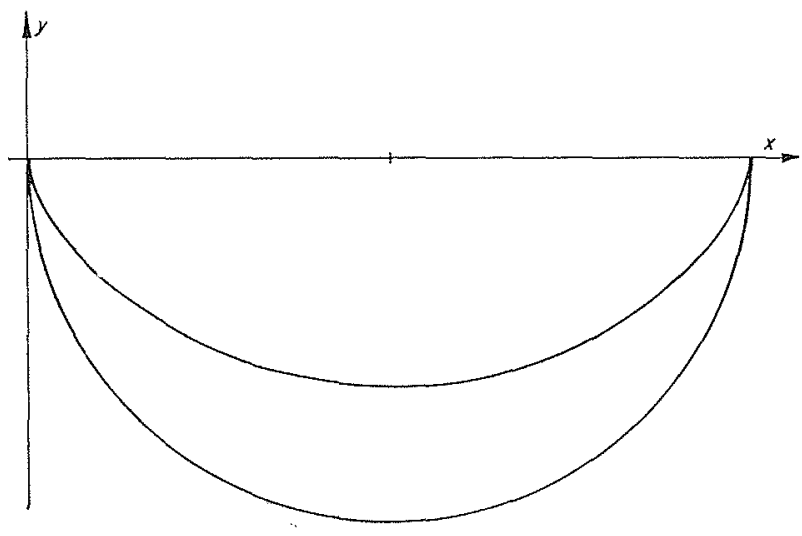

FIG. 30

La courbe - 1/Ф (A) est alors une droite passant par le point - 1 (fig. 31). Le domaine interdit est hachuré sur la figure 31 .

Ce domaine ne dépend pas de la grandeur du jeu.

Etudions simplement le cas de l'installation 


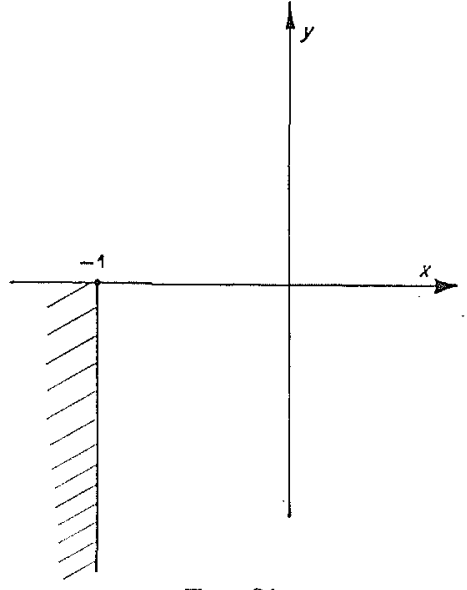

FyG. 31

figure 11. La fonction de transfert de la partie linéaire est :

$$
\mathbf{F}(j \omega)=\frac{1}{\mathrm{~S} j \omega} \frac{\mathbf{K}_{0}}{j \omega+\mathbf{K}_{0} m} \frac{1}{2+\Theta j \omega} \frac{2 \mathrm{Q}}{\mathrm{Y}}
$$

La condition est ici simplement :

$$
\text { 凡 }[\mathrm{F}(j \omega)]>-1
$$

$\Re[\mathrm{F}(j \omega)]=\frac{-\left(2+\mathrm{K}_{0} m \Theta\right)}{\left(\omega^{2}+\mathrm{K}_{0}{ }^{2} m^{2}\right)\left(4+\Theta^{2} \omega^{2}\right)} \frac{2 \mathrm{Q} \mathrm{K}_{0}}{\mathrm{~S} \mathbf{Y}}$

D'où :

$\left.2+\mathrm{K}_{0} m \Theta<\left(\omega^{2}+\mathbf{K}_{0}{ }^{2} m^{2}\right)\left(4+\Theta^{2}{ }^{2}\right)^{2}\right) \frac{S Y}{2 Q K_{0}}$

La fréquence la plus défavorable est : $\omega=0$

On trouve :

$$
\frac{2 \mathrm{~S} m \mathrm{Y}}{\mathrm{Q} \Theta}>1+\frac{2}{\mathrm{~K}_{0} m \Theta}
$$

Si on ne tient pas compte du jeu, la condition de stabilité est :

$$
\frac{2 \mathrm{~S} m \mathrm{Y}}{\mathrm{Q} \Theta}>\frac{1}{1+(1 / 2) \mathbf{K}_{0} m \Theta}
$$

Les deux conditions sont représentées par les courbes de la figure 32 .

On voit que le jeu réduit très considérablement le domaine de stabilité.

Comme on a vu, ni condition de stabilité ni fréquence du cycle limite éventuel ne dépendent de la grandeur du jeu, seule l'amplitude du cycle limite lui est proportionnelle. Comme toutes les installations ont un jeu quelque part (si petit soit-il), il est bon de satisfaire à la condition de stabilité avec jeu si on veut éviter les pompages même de faible amplitude (par exemple pour des raisons mécaniques). On voit donc ici une nouvelle raison pour laquelle les courbes de transfert des boucles doivent passer loin du point - 1 (cf. fig. 29).

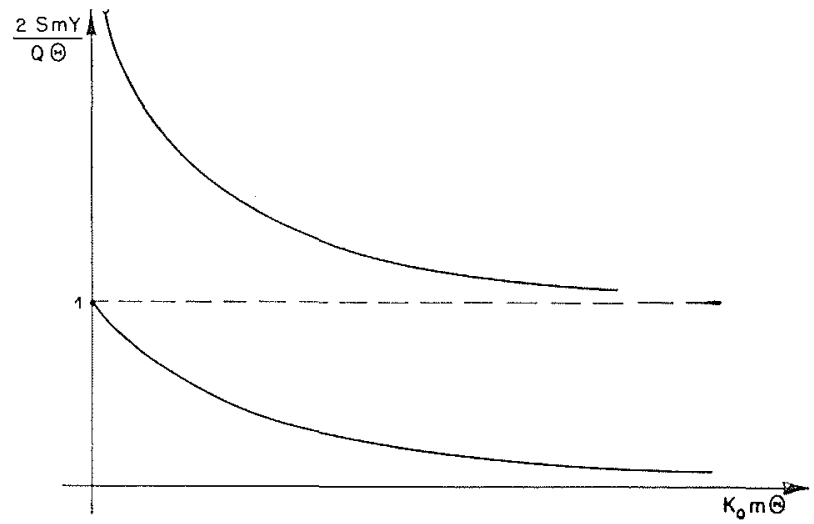

FIG. 32. - II y a stabilité à droite vers le haut. La courbe inférieure correspond à la limite de stabilité compte non tenu du jeu, la courbe supéricure à cette méme limite, compte tenu du jeu.

\section{VII. - RESUME FINAL ET CONCLUSIONS.}

Le but de notre étude était d'exposer une méthode permettant d'étudier les installations réglées automatiquement et contenant des éléments à caractéristiques non linéaires. On sait que de telles installations ne pouvaient pas être étudiées jusqu'à présent. Non seulement on ne savait pas étudier une installation numériquement déterminée, mais surtout (et ceci est encore bien plus grave) on ne savait pas quelle influence pouvait avoir les non-linéarités sur cette installation.

En pratique, aucune installation n'est rigoureusement linéaire. Si l'on ignore l'influence des non-linéarités, il est impossible de discerner l'erreur que l'on commet en les négligeant. Ainsi, nous avons montré au \& VI-23 que, dans certains cas, un jeu, apparemment négligeable a priori, peut avoir un effet considérable et être un des facteurs principaux de l'instabilité. Le fait est que les non-linéarités sont parfois négligeables; dans ce cas, la connaiśsance d'une théorie des éléments non linéaires ne modifiera en rien la méthode classique; mais on sera sûr que cette méthode est valable. Dans les autres cas, on sera obligé de faire une étude moins schémati- 
que. Jusqu'à présent, on avait l'habitude de linéariser automaliquement toutes les installations. Ceci était tout à fait normal puisqu'on ne savait pas faire autrement.

Après cette schématisation souvent extrêmement poussée, on ne s'arrêtait malheureusement pas toujours aux calculs relativement simples qui donnent des idées générales malgré tout assez exactes. De ce fait, la régulation avait de plus en plus tendance à devenir une branche des mathématiques appliquées avec tous les dangers que cela comporte.

Il semble donc que l'on ait intérèt à reprendre le problème à l'origine et à retourner aux bases physiques. On se rend alors compte qu'on ne connaît jamais exactement l'équation régissant une installation ou un appareil. On connaît au mieux un certain nombre d'équations, chacune valable approximativement dans un domaine de fonctionnement donné. Fn fait, ce domaine dépend de beaucoup de paramètres; en pratique, - pour le mode de fonctionnement courant, - il suffit de connaître les valeurs de certaines variables et leurs dérivées premières que l'on traduit par des amplitudes et des fréquences. Les frontières des domaines envisagés dépendent des erreurs que l'on consent à tolérer, et, d'une façon plus générale, des connaissances que l'on possède sur l'appareil à étudier.

Vue sous cet angle, l'étude d'un appareil paraît se ramener à celle d'un problème mathématique extrêmement compliqué. Mais si l'on ne perd pas de vue le caractère obligatoirement approché de l'équation de base, on se trouve amené à chercher une méthode d'étude simple, mais conservant à l'approximation son ordre de grandeur. Nous avons montré que les propriétés des installations réglées automatiquement peuvent en général être schématisées par un ou plusieurs ríseaux de courbe relativement faciles à obtenir et à manipuler. En général, un seul réseau de courbes permet déjà une étude beaucoup plus exacte que la linéarisation. La linéarisation apparait comme une schématisation extrêmement poussée : chaque réseau de courbe y est remplacé par une seule courbe, toutes les courbes obtenues ainsi se déduisent obligatoirement du choix de l'une d'entre elles dont la forme n'est, de plus, pas entièrement arbitraire. Souvent on est obligé de choisir une courbe qui ne se trouve pas dans la zone du plan balayce par les courbes plus exactes.

La méthode exposće iei permet done de remplacer la schématisation appeléc linéarisation par une autre schématisation au moins aussi précise et dont le degré d'approximation neut etre choisi à l'avance avec une grande latitude. Le degré de complexité de l'étude faite à propos d'une instal- lation dépend évidemment de la précision que l'on désire obtenir. De toute façon on peut s'arlanger pour que les études mathématiques ne soient pas disproportionnées par rapport à la schématisation physique.

Le principe fondamental sur lequel repose la méthode exposée ici est le suivant :

- Le spectre de Fourier d'un mouvement oscillatoire stationnaire ou lentement variable d'une installation réglée automatiquement possède une pointe très marquée et est à peu près nul partout ailleurs. Plus simplement, on peut dire que ces installations sont des filtres passebas, ce qui est bien conmu. La méthode de Nyourst se généralise alors facilement. Rappelons qu'elle consiste, pour les installations linéaires, à ouvrir la boucle formée par l'instalIation et à la soumettre à des excitations sinusoüdales de différentes fréquences. Connaissant les réponses à ces excitations, on en déduit toutes les propriétés de l'installation. Nous avons montré que la même méthode pouvait être appliquée aux installations non linéaires à condition de tenir compte de l'amplitude des excitations et, eventuellement, des variations de l'amplitude et de la fréquence. Cette étude donne alors des renseignements sur la stabilité et le comportement des installations.

La connaissance du réseau de courbes de réponse à amplitude et à fréquence constantes permet de trouver les cycles limites, done les domaines de stabilité. Cette étude est parfois suffisante. Mais il n'y a évidemment aucune raison pour que, de ce réseau de courbes, on puisse déduire les mouvements lentement variables de l'installation. Si l'on veut analyser ces mouvements, il faut connaître plusieurs autres réseaux de courbes de réponse. Ainsi, il faut trois réseaux de courbes pour étudier les régimes lentement variables et la stabilité des cycles limites. On voit qu'on est amené à faire une analyse harmonique de plus en plus poussée. On peut aussi étudier les réponses aux excitations ayant un certain spectre de Fourrer (ne se réduisant pas à une pointe infiniment mince, comme c'est le cas d'une sinusö̈de pure). L'intérêt de la méthode préconisée ici réside dans le fait qu'elle permet de faire une étude, peut-être incomplète, mais techniquement amplement suffisante, avec relativement neu de calcul. Elle permet l'étude des phénomènes courants d'une facon simple, donc phrsicfuement et intuitivemeni compréhensible. Elle permet aussi, de ce fait, de dégager des principes généraux simples traduisant les pronriétés des principaux dispositifs que l'on pent emplover ou rencontrer, et d'accroître ainsi l'intuition indépendamment de toute considération mathématique. C'est ce dernier point que 
nous considérons comme de loin le plus important. Ainsi les propriétés des transmissions à jeu indiquées brièvement aux \$ V-232; VI-11 et VII23 et les propriétés analogues d'autres dispositifs qu'on peut explorer de la mème manière aident a dégrossir les problèmes de régulation et à fixer les grandes lignes du régulateur à adopter pour telle ou telle installation.

Au terme de cette trés longue étude, nous nous faisons un devoir de rendre hommage à ceux qui, a notre connaissance, ont apporté une contribution directe à la solution de ces problèmes.

D'après les recherches bibliographiques auxquelles nous nous sommes livrés dans ce domaine, il semble bien que W. OpPeLT soit le premier à avoir, dans son livre [8], publié en 1947, émis l'idée d'une analyse harmonique des systèmes non linéaires. En particulier, il montrait que l'injection d'une excitation sinusoüdale dans une certaine classe d'éléments non linéaires - nous les aṽons qualifiés « d'algébriques»-- provoque une réponse périodique et que l'on peut tracer une courbe de Nyoust en fonction de l'amplitude du signal injecté.

Plus récemment, nous l'avons d'ailleuts signalé dans le corps de notre étude, MM. KocHerBURger et DuTruh ont publié séparément [3] [4] une méthode permettant l'étude partielle des systèmes comprenant uniquement des éléments linéaires et algébriques. Enfin, depuis 1951, on doit à M. LoEB de nombreuses publications, étendant la méthode à tous les systèmes non linéaires et proposant une théorie de la stabilité des cycles limites assez différente de la nôtre.

Nous espérons, quant a nous, avoir fait bénéficier cette méthode de quelques apports substantiels. Nous espérons également que certaines des controverses que nous soulevons s'avéreront fructueuses. Nous sommes heureux enfin d'anoir aidé a la diffusion de ces procédés parmi les Hydrauliciens qui, dans ce domaine de la régulation, se tronvent aux prises avec des problèmes dépassant peut-être, en portée et en complexité, ceux qui ont provoqué les recherches antérieures.

\section{BIBLIOGRAPHIE}

1.-- BAYARD : Relations entre les parties réelles et imaginaires des impédances. R.G.E., mai 1935, p. 659 .

2. - Bergeron: Du coup de béliex au coup de foudre. Editeur : Dunod, Paris, 1950

3. - Dutrly : Théorie des servo-mécanismes à relais. Onde électrique, octobre 1950, p. 438.

4. -- Kochendurger : Analysis contactor servo-mechanisms. Electrical Engineering, avril 1950.

5. - Kuntzanne, Daniel, Ma Min Yuan : Stabilité des systèmes de réglage. R.G.E., mars 1952 , p. 149 .
6. - Lozm : C. R. Académie des Sciences, t. 233, p. 344.

- Phénomènes héréditaires dans les servo-mécanismes. Annales des Télécommunications. Déc. 1951.

- Transitoires dans les servo-mécanismes filtrés. Annales des Télécommunications. Oct. 1952.

7. - R. Meyer : Quelques résultats nouveaux concernant la régulation des turbines et des installations hydrauliques en général.

La Houtlle Blanche, no 4-1952.

8. - W. Oppezt : Grundgesetze der Reglung. Editeur : Wolfbiutler Verlags instalt, 1947. 